\title{
A Mathematical Model for Solving the Linear Programming Problems Involving Trapezoidal Fuzzy Numbers via Interval Linear Programming Problems
}

\author{
Ladji Kané (iD, Daouda Diawara, Lassina Diabaté, Moussa Konaté, Souleymane Kané, \\ and Hawa Bado
}

Department of Applied Mathematics (FSEG), Université des Sciences Sociales et de Gestion de Bamako (USSGB), Quartier du Fleuve Rue 310, Porte 238, Mali

Correspondence should be addressed to Ladji Kané; fsegmath@gmail.com

Received 6 February 2021; Revised 15 March 2021; Accepted 17 March 2021; Published 17 April 2021

Academic Editor: Xiaolong Qin

Copyright (C) 2021 Ladji Kané et al. This is an open access article distributed under the Creative Commons Attribution License, which permits unrestricted use, distribution, and reproduction in any medium, provided the original work is properly cited.

We define linear programming problems involving trapezoidal fuzzy numbers (LPTra) as the way of linear programming problems involving interval numbers (LPIn). We will discuss the solution concepts of primal and dual linear programming problems involving trapezoidal fuzzy numbers (LPTra) by converting them into two linear programming problems involving interval numbers (LPIn). By introducing new arithmetic operations between interval numbers and fuzzy numbers, we will check that both primal and dual problems have optimal solutions and the two optimal values are equal. Also, both optimal solutions obey the strong duality theorem and complementary slackness theorem. Furthermore, for illustration, some numerical examples are used to demonstrate the correctness and usefulness of the proposed method. The proposed algorithm is flexible, easy, and reasonable.

\section{Introduction}

In the real-world environment, there are many problems which are a concern to the linear programming models, and sometimes it is necessary to formulate these models with parameters of uncertainty. Many numbers from these problems are linear programming problems with fuzzy variables. Interval analysis is an efficient and reliable tool that allows us to handle such problems effectively.

Linear programming problems with interval coefficients have been studied by several authors, such as Sengupta et al. [1, 2], Bitran [3], Chanas and Kuchta [4], Nakahara et al. [5], Steuer [6], and Shaocheng [7]. Numerous methods for comparison of interval numbers can be found as in Sengupta et al. [1, 2], Ganesan and Veeramani [8, 9], etc. By taking maximum value range and minimum value range inequalities as constraint conditions, Shaocheng [7] reduced the interval linear programming problem into two classical linear programming problems and obtained an optimal interval solution to it. Ramesh and Ganesan [10] proposed a method for solving interval number linear programming problems without converting them to classical linear programming problems.

Bellman and Zadeh [11] introduced for the first time the concept of a fuzzy decision process as an intersection of the fuzzy objective function and resource constraints. From this idea, the authors of [12-20] introduced linear programming problems with fuzzy variables and semifully fuzzy linear programming problems. Some authors considered these problems and have developed various methods for solving these problems. Recently, some authors [12-20] considered linear programming problems with trapezoidal fuzzy data and/or variables and semifully fuzzy linear programming problems and stated a fuzzy simplex algorithm to solve these problems. Moreover, they developed the duality results in fuzzy environment and presented a dual simplex algorithm 
for solving linear programming problems with trapezoidal fuzzy variables and semifully fuzzy linear programming problems. Furthermore, the authors of [12-20] showed that the presented dual simplex algorithm directly using the primal simplex tableau algorithm tenders the capability for sensitivity (or postoptimality) analysis using primal simplex tableaus.

The contributions of the present study are summarized as follows. (1) In the LPTra under consideration, all of the parameters, such as the coefficients in the objective function, the right-hand side vector, and the decision variables, are a kind of trapezoidal fuzzy numbers, simultaneity. Furthermore, the coefficients in the left-hand side matrix are symmetric trapezoidal fuzzy numbers. (2) According to the proposed approach, the LPTra is converted into two linear programming problems involving interval numbers. The integration of the optimal solution of the two subproblems provides the optimal solution of the primary LPTra. (3) In contrast to most existing approaches, which provide a precise solution, the proposed method provides a fuzzy optimal solution. (4) Similarly, to the competing methods in the literature, the proposed method is applicable for all types of real problems. (5) The complexity of computation is greatly reduced compared with commonly used existing methods in the literature.

The rest of this paper is organized as follows. In Section 2 , we recall the definitions of interval number linear programming, interval numbers, and existing method for solving linear programming problem involving interval numbers. In Section 3, a new method is proposed for obtaining the fuzzy optimal solution of the LPTra. The advantages of the proposed method are discussed in Section 4. Two application examples are provided to illustrate the effectiveness of the proposed method in Section 5. Comparative study is presented in Section 6. Finally, concluding remarks are presented in Section 7.

\section{Materials and Methods}

In this section, some basic definitions and arithmetic operations for closed intervals' numbers and of linear programming problems involving interval numbers are presented [21-23].

\subsection{Arithmetic Operations for Closed Intervals' Numbers.} In this section, some arithmetic operations for two intervals are presented $[22,24]$.

Let us denote by $\mathfrak{T}$ the class of all closed intervals in $\mathbb{R}$. If $A$ is closed interval, we also adopt the notation $\bar{a}=\left[a^{1}, a^{4}\right]$, where $a^{1}$ and $a^{4}$ mean the lower and upper bounds of $\bar{a}$, respectively, and $a^{1} \leq a^{4}$ with $a^{1}, a^{4} \in \mathbb{R}$.

If $\bar{a}=a^{1}=a^{4}=a$, then $\bar{a}=[a, a]=a$. Any two intervals $\bar{a}=\left[a^{1}, a^{4}\right]$ and $\bar{b}=\left[b^{1}, b^{4}\right]$ and the arithmetic operations on $\bar{a}$ and $\bar{b}$ are defined as (i) Addition: $\quad \bar{a}+\bar{b}=\left[a^{1}+b^{1}, a^{4}+b^{4}\right]$, subtraction: $\bar{a}-\bar{b}=\left[a^{1}-b^{4}, a^{4}-b^{1}\right]$

Multiplication: $\quad \bar{a} \times \bar{b}=\left[\operatorname{Min}\left\{a^{1} \times b^{1}, a^{1} \times b^{4}, a^{4} \times b^{1}\right.\right.$, $\left.\left.a^{4} \times b^{4}\right\}, \operatorname{Max}\left\{a^{1} \times b^{1}, a^{1} \times b^{4}, a^{4} \times b^{1}, a^{4} \times b^{4}\right\}\right]$

Division: $\quad \bar{a} \div \bar{b}=\left[\operatorname{Min}\left\{\left(a^{1} / b^{4}\right),\left(a^{1} / b^{1}\right),\left(a^{4} / b^{4}\right),\left(a^{4}\right.\right.\right.$ $\left.\left.\left./ b^{1}\right)\right\}, \quad \operatorname{Max}\left\{\left(a^{1} / b^{4}\right),\left(a^{1} / b^{1}\right),\left(a^{4} / b^{4}\right),\left(a^{4} / b^{1}\right)\right\}\right]$ with $0 \notin \bar{b}$

2.2. A New Interval Arithmetic. In this section, some arithmetic operations for two intervals are presented [21, 23].

Let $\mathfrak{R}=\left\{\bar{a}^{14}=\left[a^{1}, a^{4}\right]: a^{1} \leq a^{4}\right.$ with $\left.a^{1}, a^{4} \in \mathbb{R}\right\}$ be the set of all proper intervals and $\overline{\mathfrak{R}}=\left\{\bar{a}^{14}=\left[a^{1}, a^{4}\right]: a^{1}>a^{4}\right.$ with $\left.a^{1}, a^{4} \in \mathbb{R}\right\}$ be the set of all improper intervals on the real line $\mathbb{R}$. We shall use the terms "interval" and "interval number" interchangeably. The midpoint and width (or half-width) of an interval number $\bar{a}^{14}=\left[a^{1}, a^{4}\right]$ are defined as $m\left(\bar{a}^{14}\right)=\left(a^{4}+a^{1} / 2\right)$ and $w\left(\bar{a}^{14}\right)=\left(a^{4}-a^{1} / 2\right)$. The interval number $\bar{a}^{14}$ can also be expressed in terms of its midpoint and width as $\bar{a}^{14}=\left[a^{1}, a^{4}\right]=m\left(\bar{a}^{14}\right)$

and $w\left(\bar{a}^{14}\right)=\left(a^{4}+a^{1} / 2\right),\left(a^{4}-a^{1} / 2\right)$.

For any two intervals $\bar{a}^{14}=\left[a^{1}, a^{4}\right]$ and $\bar{b}^{14}=\left[b^{1}, b^{4}\right]$, the arithmetic operations on $\bar{a}^{14}$ and $\bar{b}^{14}$ are defined as

(i) Addition: $\quad \bar{a}^{14}+\bar{b}^{14}=\left[a^{1}, a^{4}\right]+\left[b^{1}, b^{4}\right]=m\left(\bar{a}^{14}\right)$ $+m\left(\bar{b}^{14}\right), \max \left\{w\left(\bar{a}^{14}\right), w\left(\bar{b}^{14}\right)\right\}$

Subtraction: $\bar{a}^{14}-\bar{b}^{14}=\left[a^{1}, a^{4}\right]-\left[b^{1}, b^{4}\right]=m\left(\bar{a}^{14}\right)-$ $m\left(\bar{b}^{14}\right), \max \left\{w\left(\bar{a}^{14}\right), w\left(\bar{b}^{14}\right)\right\}$

Multiplication: $\quad \bar{a}^{14} \times \bar{b}^{14}=\left[a^{1}, a^{4}\right] \times\left[b^{1}, b^{4}\right]=$ $m\left(\bar{a}^{14}\right) \times m\left(\bar{b}^{14}\right), \max \left\{w\left(\bar{a}^{14}\right), w\left(\bar{b}^{14}\right)\right\}$

Division: $\quad \bar{a}^{14} \div \bar{b}^{14}=\left[a^{1}, a^{4}\right] \div\left[b^{1}, b^{4}\right]=m\left(\bar{a}^{14}\right) \div$ $m\left(\bar{b}^{14}\right), \max \left\{w\left(\bar{a}^{14}\right), w\left(\bar{b}^{14}\right)\right\}$ with $m\left(\bar{b}^{14}\right) \approx 0$

2.3. Ramesh and Ganesan's Method for Solving Linear Programming Problem Involving Interval Numbers. In this section, to overcome all the limitations of the method presented in [22], Ramesh and Ganesan's method [21] is presented to find the exact optimal solution of linear programming problems involving interval numbers in which all the parameters are represented by intervals numbers.

2.3.1. Formulation of a Linear Programming Problem Involving Interval Numbers (LPIn). Now, we are in a position to prove interval analogue of some important relationships between the primal and dual linear programming problems.

We consider the primal $(P)$ and dual $(D)$ linear programming problems involving interval numbers (LPIn) as follows [21-23]: 

$(P) \begin{cases}\operatorname{Max} & \bar{Z}(\bar{x}) \approx \sum_{j=1}^{n} \bar{c}_{j} \bar{x}_{j} \\ \text { Subject to the constraints } & \sum_{j=1}^{n} \bar{a}_{i j} \bar{x}_{j} \circ \bar{b}_{i},\end{cases}$
(D) $\begin{cases}\text { Min } & \bar{W}(\bar{y}) \approx \sum_{i=1}^{m} \bar{b}_{i} \bar{y} \\ \text { Subject to the constraints } & \sum_{i=1}^{m} \bar{a}_{i j} \bar{y}_{i} \pm \bar{c}_{j} .\end{cases}$

For the rest of this paper, we will consider the following notations:

$$
\begin{aligned}
& \bar{x}^{14}=\left[\bar{x}_{j}^{14}\right]_{n \times 1}=\left[\left[x_{j}^{1}, x_{j}^{4}\right]\right]_{n \times 1}=\left[m\left(\bar{x}_{j}^{14}\right), w\left(\bar{x}_{j}^{14}\right)\right]_{n \times 1}, \\
& \bar{x}^{23}=\left[\bar{x}_{j}^{23}\right]_{n \times 1}=\left[\left[x_{j}^{2}, x_{j}^{3}\right]\right]_{n \times 1}=\left[m\left(\bar{x}_{j}^{23}\right), w\left(\bar{x}_{j}^{23}\right)\right]_{n \times 1}, \\
& \bar{c}^{14}=\left[\bar{c}_{j}^{14}\right]_{1 \times n}=\left[\left[c_{j}^{1}, c_{j}^{4}\right]\right]_{1 \times n}=\left[m\left(\bar{c}_{j}^{14}\right), w\left(\bar{c}_{j}^{14}\right)\right]_{1 \times n}{ }^{2} \\
& \bar{c}^{23}=\left[\bar{c}_{j}^{23}\right]_{1 \times n}=\left[\left[c_{j}^{2}, c_{j}^{3}\right]\right]_{1 \times n}=\left[m\left(\bar{c}_{j}^{23}\right), w\left(\bar{c}_{j}^{23}\right)\right]_{1 \times n}, \\
& \bar{b}^{14}=\left[\bar{b}_{i}^{14}\right]_{m \times 1}=\left[\left[b_{i}^{1}, b_{i}^{4}\right]\right]_{m \times 1}=\left[m\left(\bar{b}_{i}^{14}\right), w\left(\bar{b}_{i}^{14}\right)\right]_{m \times 1}, \\
& \bar{b}^{23}=\left[\bar{b}_{i}^{23}\right]_{m \times 1}=\left[\left[b_{i}^{2}, b_{i}^{3}\right]\right]_{m \times 1}=\left[m\left(\bar{b}_{i}^{23}\right), w\left(\bar{b}_{i}^{23}\right)\right]_{m \times 1}, \\
& \bar{A}^{14}=\left[\bar{a}_{i j}^{14}\right]_{m \times 1}=\left[\left[a_{i j}^{1}, a_{i j}^{4}\right]\right]_{m \times 1}=\left[m\left(\bar{a}_{i j}^{14}\right), w\left(\bar{a}_{i j}^{14}\right)\right]_{m \times 1}, \\
& \bar{A}^{23}=\left[\bar{a}_{i j}^{23}\right]_{m \times 1}=\left[\left[a_{i j}^{2}, a_{i j}^{3}\right]\right]_{m \times 1}=\left[m\left(\bar{a}_{i j}^{23}\right), w\left(\bar{a}_{i j}^{23}\right)\right]_{m \times 1},
\end{aligned}
$$

where $x_{j}^{1}, x_{j}^{4}, c_{j}^{1}, c_{j}^{4}, b_{i}^{1}, b_{i}^{4}, x_{j}^{2}, x_{j}^{3}, c_{j}^{2}, c_{j}^{3}, b_{i}^{2}, b_{i}^{3}, \bar{a}_{i j}^{14}$, and $\bar{a}_{i j}^{23}$ are real numbers $(\mathbb{R})$.
For the rest of this paper, we will consider the following primal linear programming problem involving interval numbers (LPIn14):

$$
\begin{cases}\frac{\operatorname{Min}}{\operatorname{Max}} & \bar{Z}^{14}\left(x_{1}^{14}, \ldots, x_{n}^{14}\right) \approx \sum_{j=1}^{n}\left[c_{j}^{1}, c_{j}^{4}\right]\left[x_{j}^{1}, x_{j}^{4}\right] \\
\text { Subject to the constraints } & \sum_{j=1}^{n}\left[a_{i j}^{1}, a_{i j}^{4}\right]\left[x_{j}^{1}, x_{j}^{4}\right]\left(\begin{array}{c}
\circ \\
\\
\pm
\end{array}\right)\left[b_{i}^{1}, b_{i}^{4}\right], 1 \leq j \leq n \text { and } 1 \leq i \leq m,\end{cases}
$$

is equivalent to




and primal linear programming problem involving interval numbers (LPIn23)

$$
\begin{cases}\frac{\operatorname{Min}}{\operatorname{Max}} & \bar{Z}^{23}\left(-^{23}, \ldots, x_{n}^{23}\right) \approx \sum_{j=1}^{n}\left[c_{j}^{2}, c_{j}^{3}\right]\left[x_{j}^{2}, x_{j}^{3}\right] \\
\text { Subject to the constraints } & \sum_{j=1}^{n}\left[a_{i j}^{2}, a_{i j}^{3}\right]\left[x_{j}^{2}, x_{j}^{3}\right]\left(\begin{array}{c}
\circ \\
\pm
\end{array}\right)\left[b_{i}^{2}, b_{i}^{3}\right], 1 \leq j \leq n \text { and } 1 \leq i \leq m,\end{cases}
$$

is equivalent to

$$
\begin{cases}\frac{\operatorname{Min}}{\operatorname{Max}} & \bar{Z}^{23}\left(\bar{x}_{1}^{23}, \ldots, \bar{x}_{n}^{23}\right) \approx \sum_{j=1}^{n} m\left(\bar{c}_{j}^{23}\right), w\left(\bar{c}_{j}^{23}\right) m\left(-x_{j}^{23}\right), w\left(-x_{j}^{23}\right) \\
\text { Subject to the constraints } & \sum_{j=1}^{n} m\left(-_{i j}^{23}\right), w\left(-\bar{a}_{i j}^{23}\right) m\left(-x_{j}^{23}\right), w\left(-x_{j}^{23}\right)\left(\begin{array}{c}
\circ \\
\pm
\end{array}\right) m\left(b_{i}^{23}\right), w\left(-b_{i}^{23}\right), 1 \leq j \leq n \text { and } 1 \leq i \leq m .\end{cases}
$$

2.3.2. Optimal Solution $\overline{\mathbf{x}}^{*}$ for Linear Programming Problem Involving Interval Numbers (LPIn). In this section, we will describe how to determine the values of the primal $(P)$ and dual $(D)$ variables.

Calculation of the values of the primal variables (LPIn):

$\bar{x}^{*} \approx\left(\begin{array}{c}\bar{x}_{1}^{*} \\ \bar{x}_{2}^{*} \\ \cdot \\ \bar{x}_{n+m}^{*}\end{array}\right)$

Assume that $\mathbf{T}$ is optimal; then, the current basis is $x_{B}^{*}=\left(\begin{array}{c}x_{J_{1}}^{*}=b_{J_{1}}^{*} \\ x_{J_{2}}^{*}=b_{J_{2}}^{*} \\ \cdot \\ x_{J_{m}}^{*}=b_{J_{m}}^{*}\end{array}\right)$. Moreover, the current nonbasic variables is $x_{N}^{*}=\left\{x_{d}^{*}\right\}$ and the corresponding solution is $x_{d}^{*}=0$. Hence, the optimal solution to the problem (LPIn) can be written as $\bar{x}_{J_{k}}^{*} \approx x_{J_{k}}^{*}, \max _{1 \leq i \leq m}\left(w\left(\bar{b}_{i}\right)\right)=\left[x_{J_{k}}^{*}-\max _{1 \leq i \leq m}\right.$ $\left.\left(w\left(\bar{b}_{i}\right)\right), x_{J_{k}}^{*}+\max _{1 \leq i \leq m}\left(w\left(\bar{b}_{i}\right)\right)\right]$ for $1 \leq k \leq m$ with the associated value of the objective function: $\bar{Z}^{*} \approx Z^{*}, \max _{1 \leq i \leq m}$ $\left(w\left(\bar{b}_{i}\right)\right)=\left[Z^{*}-\max _{1 \leq i \leq m}\left(w\left(\bar{b}_{i}\right)\right), Z^{*}+\max _{1 \leq i \leq m}\left(w\left(\bar{b}_{i}\right)\right)\right]$ or $\bar{Z}\left(\bar{x}^{*}\right) \approx \bar{c} \bar{x}^{*}=\left[Z^{* 1}, Z^{* 4}\right]$.

Calculation of the values of the dual $(D)$ variables $\bar{y}^{*} \approx\left(\bar{y}_{1}^{*} \bar{y}_{2}^{*} \ldots \bar{y}_{n+m}^{*}\right)$ in $\bar{T}$.
We have $\bar{y}_{i}^{*} \approx\left|\Delta_{n+i}\right|, \max _{1 \leq j \leq n}\left(w\left(\bar{c}_{j}\right)\right)=\left[\left|\Delta_{n+i}\right|-\right.$ $\left.\max _{1 \leq j \leq n}\left(w\left(\bar{c}_{j}\right)\right),\left|\Delta_{n+i}\right|+\max _{1 \leq j \leq n}\left(w\left(\bar{c}_{j}\right)\right)\right]$ and $\bar{y}_{m+j}^{*} \approx\left|\Delta_{j}\right|$, $\max _{1 \leq j \leq n}\left(w\left(\bar{c}_{j}\right)\right)=\left[\left|\Delta_{j}\right|-\max _{1 \leq j \leq n}\left(w\left(\bar{c}_{j}\right)\right),\left|\Delta_{j}\right|+\right.$ $\left.\max _{1 \leq j \leq n}\left(w\left(\bar{c}_{j}\right)\right)\right]$ with the associated value of the objective function: $\quad \bar{W}^{*} \approx W^{*}, \max _{1 \leq j \leq n}\left(w\left(\bar{c}_{j}\right)\right)=\left[W^{*}-\max _{1 \leq j \leq n}\right.$ $\left.\left(w\left(\bar{c}_{j}\right)\right), W^{*}+\max _{1 \leq j \leq n}\left(w\left(\bar{c}_{j}\right)\right)\right]$ $\operatorname{Min} \bar{W}\left(\bar{y}^{*}\right) \approx \bar{y}^{*} \bar{b}=\left[W^{* 1}, W^{* 4}\right]$.

\section{Main Results}

In this section, we will describe our method of solving.

3.1. A New Interval Arithmetic for Trapezoidal Fuzzy Numbers via Intervals' Numbers. The aim of this section is to present some notations, notions, and results which are useful for our further consideration.

A number $\tilde{a}=\left(a^{1}, a^{2}, a^{3}, a^{4}\right)$ (where $\left.a^{1} \leq a^{2} \leq a^{3} \leq a^{4}\right)$ is said to be a trapezoidal fuzzy number if its membership function is given by $[12,24,25]$

$$
\mu_{a}(x)= \begin{cases}\frac{x-a^{1}}{a^{2}-a^{1}}, & a^{1} \leq x \leq a^{2}, \\ \frac{x-a^{4}}{a^{3}-a^{4}}, & a^{3} \leq x \leq a^{4} .\end{cases}
$$

Assume that $\tilde{a}=\left(a^{1}, a^{2}, a^{3}, a^{4}\right)=\left(\bar{a}^{14} \mid \bar{a}^{23}\right)=\left(\left[a^{1}, a^{4}\right] \mid\right.$ $\left.\left[a^{2}, a^{3}\right]\right)$ and $\widetilde{b}=\left(b^{1}, b^{2}, b^{3}, b^{4}\right)=\left(\bar{b}^{14} \mid \bar{b}^{23}\right)=\left(\left[b^{1}, b^{4}\right] \mid\left[b^{2}\right.\right.$, 
$\left.b^{3}\right]$ ) are two trapezoidal fuzzy numbers. For any two trapezoidal fuzzy numbers $\widetilde{a}=\left(\bar{a}^{14} \mid \bar{a}^{23}\right)$ and $\widetilde{b}=\left(\bar{b}^{14} \mid \bar{b}^{23}\right)$, the arithmetic operations on $\widetilde{a}$ and $\widetilde{b}$ are defined as follows.

Addition:

$$
\begin{aligned}
\widetilde{a}+\widetilde{b}= & \left(\bar{a}^{14} \mid \bar{a}^{23}\right)+\left(\bar{b}^{14} \mid \bar{b}^{23}\right)=\left(\left[a^{1}, a^{4}\right]+\left[b^{1}, b^{4}\right] \mid\left[a^{2}, a^{3}\right]\right. \\
& \left.+\left[b^{2}, b^{3}\right]\right) .
\end{aligned}
$$

Subtraction:

$$
\begin{aligned}
\widetilde{a}-\widetilde{b}= & \left(\bar{a}^{14} \mid \bar{a}^{23}\right)-\left(\bar{b}^{14} \mid \bar{b}^{23}\right)=\left(\left[a^{1}, a^{4}\right]-\left[b^{1}, b^{4}\right] \mid\left[a^{2}, a^{3}\right]\right. \\
& \left.-\left[b^{2}, b^{3}\right]\right) .
\end{aligned}
$$

Multiplication:

$$
\begin{aligned}
\tilde{a} \times \tilde{b}= & \left(\bar{a}^{14} \mid \bar{a}^{23}\right) \times\left(\bar{b}^{14} \mid \bar{b}^{23}\right)=\left(\left[a^{1}, a^{4}\right] \times\left[b^{1}, b^{4}\right] \mid\left[a^{2}, a^{3}\right]\right. \\
& \left.\times\left[b^{2}, b^{3}\right]\right) .
\end{aligned}
$$

For the rest of this paper, we will consider the following notations.

Assume that $\tilde{c}_{j}=\left(c_{j}^{1}, c_{j}^{2}, c_{j}^{3}, c_{j}^{4}\right), \tilde{x}_{j}=\left(x_{j}^{1}, x_{j}^{2}, x_{j}^{3}, x_{j}^{4}\right)$, and $\widetilde{b}_{i}=\left(b_{i}^{1}, b_{i}^{2}, b_{i}^{3}, b_{i}^{4}\right)$ are trapezoidal fuzzy numbers:

$$
\begin{aligned}
& \tilde{x}=\left[\widetilde{x}_{j}\right]_{n \times 1}=\left[\left(\bar{x}_{j}^{14} \mid \bar{x}_{j}^{23}\right)\right]_{n \times 1}=\left[\left(m\left(\bar{x}_{j}^{14}\right), w\left(\bar{x}_{j}^{14}\right) \mid m\left(\bar{x}_{j}^{23}\right), w\left(\bar{x}_{j}^{23}\right)\right)\right]_{n \times 1}, \\
& \widetilde{c}=\left[\widetilde{c}_{j}\right]_{1 \times n}=\left[\left(\bar{c}_{j}^{14} \mid \bar{c}_{j}^{23}\right)\right]_{1 \times n}=\left[\left(m\left(\bar{c}_{j}^{14}\right), w\left(\bar{c}_{j}^{14}\right) \mid m\left(\bar{c}_{j}^{23}\right), w\left(\bar{c}_{j}^{23}\right)\right)\right]_{1 \times n}, \\
& \widetilde{b}=\left[\widetilde{b}_{i}\right]_{m \times 1}=\left[\left(\bar{b}_{i}^{14} \mid \bar{b}_{i}^{23}\right)\right]_{m \times 1}=\left[\left(m\left(\bar{b}_{i}^{14}\right), w\left(\bar{b}_{i}^{14}\right) \mid m\left(\bar{b}_{i}^{23}\right), w\left(\bar{b}_{i}^{23}\right)\right)\right]_{m \times 1}, \\
& \widetilde{A}=\left[\widetilde{a}_{i j}\right]_{m \times n}=\left[\left(\bar{a}_{i j}^{14} \mid \bar{a}_{i j}^{23}\right)\right]_{m \times n}=\left[\left(m\left(\bar{a}_{i j}^{14}\right), w\left(\bar{a}_{i j}^{14}\right) \mid m\left(\bar{a}_{i j}^{23}\right), w\left(\bar{a}_{i j}^{23}\right)\right)\right]_{m \times n} .
\end{aligned}
$$

where $x_{j}^{1}, x_{j}^{4}, c_{j}^{1}, c_{j}^{4}, b_{i}^{1}, b_{i}^{4}, x_{j}^{2}, x_{j}^{3}, c_{j}^{2}, c_{j}^{3}, b_{i}^{2}, b_{i}^{3}, \bar{a}_{i j}^{14}$, and $\bar{a}_{i j}^{23}$ are real numbers $(\mathbb{R})$.

\subsection{Formulation of Linear Programming Problems Involving} Trapezoidal Fuzzy Numbers. In this section, we introduce a kind of linear programming problems where the coefficients in objective function, the right-hand-side vector, and the decision variables are a type of trapezoidal fuzzy numbers, simultaneity, and the left-hand-side matrix having symmetric trapezoidal fuzzy numbers coefficients. We name such problems as linear programming problems involving trapezoidal fuzzy numbers (LPTra).

We consider the primal $(P)$ and dual $(D)$ linear programming problems involving trapezoidal fuzzy numbers (LPTra) as follows [12, 25]:
$(P) \begin{cases}\operatorname{Max} & \widetilde{Z}(\widetilde{x}) \approx \sum_{j=1}^{n} \widetilde{c}_{j} \widetilde{x}_{j}, \\ \text { Subject to the constraints } & \sum_{j=1}^{n} \widetilde{a}_{i j} \widetilde{x}_{j} \circ \widetilde{b}_{i}, \widetilde{a}_{i j}: \text { symmetric trapezoidal fuzzy numbers, } 1 \leq j \leq n, 1 \leq i \leq m,\end{cases}$
$\begin{cases}\text { Min } & \tilde{W}(\tilde{y}) \approx \sum_{i=1}^{m} \widetilde{b}_{i} \widetilde{y}_{i}, \\ \text { Subject to the constraints } & \sum_{i=1}^{m} \widetilde{a}_{i j} \tilde{y}_{i} \succcurlyeq \widetilde{c}_{j} .\end{cases}$ 
For the rest of this paper, we will consider the following primal linear programming problems involving trapezoidal fuzzy numbers (LPTra):

$$
\begin{aligned}
& \frac{\operatorname{Min}}{\operatorname{Max}} \\
& \widetilde{Z}\left(\tilde{x}_{1}, \ldots, \tilde{x}_{n}\right) \approx \sum_{j=1}^{n}\left(\bar{c}_{j}^{14} \mid \bar{c}_{j}^{23}\right)\left(x_{j}^{14}\left\lceil x_{j}^{23}\right)\right. \\
& \sum_{j=1}^{n}\left({ } ^ { 1 4 } a _ { i j } \lceil a _ { i j } ^ { 2 3 } ) \left(x_{j}^{14}\left\lceil x_{j}^{23}\right)\left(\begin{array}{l}
\preccurlyeq \\
\succcurlyeq
\end{array}\right)\left(b_{i}^{14} \mid b_{i}^{23}\right),\right.\right. \\
& x_{j}^{1}+\min _{1 \leq i \leq m}\left(w\left(\bar{b}_{i}\right)\right) \leq m\left(x_{j}^{23}\right) \leq x_{j}^{4}-\min _{1 \leq i \leq m}\left(w\left(\bar{b}_{i}\right)\right) \\
& \left({ }^{-} a_{i j} \mid{ }^{14} a_{i j}^{23}\right): \text { symmetric trapezoidal fuzzy numbers, } 1 \leq j \leq n \text { and } 1 \leq i \leq m \text {. }
\end{aligned}
$$

3.3. Our Method for Solving the Linear Programming Problems Involving Trapezoidal Fuzzy Numbers (LPTra). In this section, a method to find a fuzzy optimal solution of linear programming problems involving trapezoidal fuzzy numbers (LPTra) is presented.
3.3.1. Formulation of a Linear Programming Problem Involving Midpoint (LPMi14). Thanks to the new interval arithmetic and (LPIn14), we can write the following linear programming problem involving midpoint (LPMi14) [21]:

$$
\begin{cases}\frac{\operatorname{Min}}{\operatorname{Max}} & Z^{14}\left(x^{14}\right)=\sum_{j=1}^{n} m\left(\bar{c}_{j}^{14}\right) x_{j}^{14}, \\
\text { Subject to the constraints } & \sum_{j=1}^{n} m\left({ }^{14} a_{i j}\right) x_{j}^{14}\left(\begin{array}{c}
\leq \\
\geq
\end{array}\right) m\left(b_{i}^{14}\right), 1 \leq j \leq n \text { and } 1 \leq i \leq m .\end{cases}
$$

3.3.2. Formulation of a Linear Programming Problem Involving Midpoint (LPMi23). Thanks to the new interval arithmetic and (LPIn23), we can write the following linear programming problem involving midpoint (LPMi23) [21]:

$$
\begin{cases}\frac{\operatorname{Min}}{\operatorname{Max}} & Z^{23}\left(x^{23}\right)=\sum_{j=1}^{n} m\left(\bar{c}_{j}^{23}\right) x_{j}^{23}, \\
\text { Subject to the constraints } & \sum_{j=1}^{n} m\left(-_{i j}^{23}\right) x_{j}^{23}\left(\begin{array}{c}
\leq \\
\geq
\end{array}\right) m\left(b_{i}^{23}\right), \\
& x_{j}^{1}+\min _{1 \leq i \leq m}\left(w\left(\bar{b}_{i}\right)\right) \leq m\left(-x_{j}^{23}\right) \leq x_{j}^{4}-\min _{1 \leq i \leq m}\left(w\left(\bar{b}_{i}\right)\right), 1 \leq j \leq n \text { and } 1 \leq i \leq m .\end{cases}
$$


Thanks to the new interval arithmetic, we can write the following lemma [21].

Lemma 1. $x^{14}=\left(x_{1}^{14}, x_{2}^{14}, \ldots, x_{n+m}^{14}\right)$ is an optimal solution to (LPMi14) if and only if $\bar{x}^{14} \approx\left(\bar{x}_{1}^{14}, \bar{x}_{2}^{14}, \ldots, \bar{x}_{n+m}^{14}\right)$ is an optimal solution to (LPIn14).

Proof (see [21]). If $x_{B}^{14}=\left(\begin{array}{c}x_{J_{1}}^{14}=b_{J_{1}}^{14} \\ x_{J_{2}}^{14}=b_{J_{2}}^{14} \\ \cdot \\ \cdot \\ x_{J_{m}}^{14}=b_{J_{m}}^{14}\end{array}\right)$ is an optimal solution to (LPMi14) and $\bar{x}_{J_{k}}^{14} \approx x_{J_{k}}^{14}, \max _{1 \leq i \leq m}\left(w\left(\bar{b}_{i}^{14}\right)\right)=\left[x_{J_{k}}^{14}-\right.$ $\left.\max _{1 \leq i \leq m}\left(w\left(\bar{b}_{i}^{14}\right)\right), x_{J_{k}}^{14}+\max _{1 \leq i \leq m}\left(w\left(\bar{b}_{i}^{14}\right)\right)\right]$ for $1 \leq k \leq m$, then $\bar{x}^{14} \approx\left(\bar{x}_{1}^{14}, \bar{x}_{2}^{14}, \ldots, \bar{x}_{n+m}^{14}\right)$ is an optimal solution to (LPIn14).

Lemma 2. $x^{23}=\left(x_{1}^{23}, x_{2}^{23}, \ldots, x_{n+m}^{23}\right)$ is an optimal solution to (LPMi23) if and only if $\bar{x}^{23} \approx\left(\bar{x}_{1}^{23}, \bar{x}_{2}^{23}, \ldots, \bar{x}_{n+m}^{23}\right)$ is an optimal solution to (LPIn23).

Proof (see [21]). If $x_{B}^{23}=\left(\begin{array}{c}x_{J_{1}}^{23}=b_{J_{1}}^{23} \\ x_{J_{2}}^{23}=b_{J_{2}}^{23} \\ \cdot \\ \cdot \\ x_{J_{m}}^{23}=b_{J_{m}}^{23}\end{array}\right)$ is an optimal solution to (LPMi23) and $\bar{x}_{J_{k}}^{23} \approx x_{J_{k}}^{23}, \min _{1 \leq i \leq m}\left(w\left(\bar{b}_{i}^{23}\right)\right)=\left[x_{J_{k}}^{23}-\right.$ $\left.\min _{1 \leq i \leq m}\left(w\left(\bar{b}_{i}^{23}\right)\right), x_{J_{k}}^{23}+\min _{1 \leq i \leq m}\left(w\left(\bar{b}_{i}^{23}\right)\right)\right]$ for $1 \leq k \leq m$, then $\bar{x}^{23} \approx\left(\bar{x}_{1}^{23}, \bar{x}_{2}^{23}, \ldots, \bar{x}_{n+m}^{23}\right)$ is an optimal solution to (LPIn23).

Thanks to the lemma above, we can write the following corollary [21].

Corollary 1. If $\bar{x}_{j}^{14} \approx\left[x_{j}^{* 1}, x_{j}^{* 4}\right]$ is an optimal solution to (LPIn14) and $\bar{x}_{j}^{23} \approx\left[x_{j}^{* 2}, x_{j}^{* 3}\right]$ is an optimal solution to (LPIn23), then $\tilde{x}^{*} \approx\left(\tilde{x}_{1}^{*} \tilde{x}_{2}^{*} \ldots \tilde{x}_{n+m}^{*}\right)^{t}$ is an optimal solution to (LPTra) with $\tilde{x}_{j}^{*} \approx\left(\bar{x}_{j}^{14} \mid \bar{x}_{j}^{23}\right)=\left(\left[x_{j}^{* 1}, x_{j}^{* 4}\right] \mid\left[x_{j}^{* 2}, x_{j}^{* 3}\right]\right)=$ $\left(x_{j}^{* 1}, x_{j}^{* 2}, x_{j}^{* 3}, x_{j}^{* 4}\right)$.

3.3.3. The Steps of Our Computational Method. The steps of our method for solving the linear programming problems involving trapezoidal fuzzy numbers (LPTra) as follows:

Step 1: consider primal and dual linear programming problems involving trapezoidal fuzzy numbers (LPTra).

Step 2: identify (LPIn14) and (LPIn23).

Step 3 (Ramesh and Ganesan's method, see [21]): solve the primal and dual (LPIn14) via (LPMi14).

Apply the simplex method to (LPMi14) to determine the primal variables (LPMi14): $x^{14}=\left(\begin{array}{c}x_{1}^{14} \\ x_{2}^{14} \\ \cdot \\ \cdot \\ x_{n+m}^{14}\end{array}\right)$. As- sume that $\mathbf{T}^{14}$ is optimal, then the current basis is $x_{B}^{14}=\left(\begin{array}{c}x_{J_{1}}^{14}=b_{J_{1}}^{14} \\ x_{J_{2}}^{14}=b_{J_{2}}^{14} \\ \cdot \\ x_{J_{m}}^{14}=b_{J_{m}}^{14}\end{array}\right)$

Moreover, the current nonbasic

variables is $x_{N}^{14}=\left\{x_{d}^{14}\right\}$ and the corresponding solution is

$x_{d}^{14}=0$. Hence, the optimal solution to problem (LPIn14) can be written as $\bar{x}_{J_{k}}^{14} \approx x_{J_{k}}^{14}, \min _{1 \leq i \leq m}\left(w\left(\bar{b}_{i}^{14}\right)\right)=\left[x_{J_{k}}^{14}-\right.$ $\left.\min _{1 \leq i \leq m}\left(w\left(\bar{b}_{i}^{14}\right)\right), x_{J_{k}}^{14}+\min _{1 \leq i \leq m}\left(w\left(\bar{b}_{i}^{14}\right)\right)\right]$ for $1 \leq k \leq m$ with the associated value of the objective function: $\bar{Z}^{14} \approx Z^{14}, \min _{1 \leq i \leq m}\left(w\left(\bar{b}_{i}^{14}\right)\right)=\left[Z^{14}-\min _{1 \leq i \leq m}\left(w\left(\bar{b}_{i}^{14}\right)\right)\right.$, $\left.Z^{14}+\min _{1 \leq i \leq m}\left(w\left(\bar{b}_{i}^{14}\right)\right)\right]$ or $\bar{Z}^{14}\left(\bar{x}^{14}\right) \approx \bar{c}^{14} \bar{x}^{14}=\left[Z^{* 1}, Z^{* 4}\right]$. Then, the corresponding dual variables $(D)$ is given by $\bar{y}^{14} \approx\left(\bar{y}_{1}^{14} \bar{y}_{2}^{14} \ldots \bar{y}_{n+m}^{14}\right)$ in $\bar{T}^{14}$. We have $\bar{y}_{i}^{14} \approx\left|\Delta_{n+i}^{14}\right|$, $\min _{1 \leq i \leq m}\left(w\left(\bar{c}_{j}^{14}\right)\right)=\left[\left|\Delta_{n+i}^{14}\right|-\min _{1 \leq i \leq m}\left(w\left(\bar{c}_{j}^{14}\right)\right),\left|\Delta_{n+i}^{14}\right|\right.$ $\left.+\min _{1 \leq i \leq m}\left(w\left(\bar{c}_{j}^{14}\right)\right)\right]$ and $\bar{y}_{m+j}^{14} \approx\left|\Delta_{j}^{14}\right|, \min _{1 \leq i \leq m}(w$ $\left.\left(\bar{c}_{j}^{14}\right)\right)=\left[\left|\Delta_{j}^{14}\right|-\min _{1 \leq i \leq m}\left(w\left(\bar{c}_{j}^{14}\right)\right),\left|\Delta_{j}^{14}\right|+\min _{1 \leq i \leq m} \quad(w\right.$ $\left.\left(\bar{c}_{j}^{14}\right)\right)$ ] with the associated value of the objective function: $\quad \bar{W}^{14} \approx W^{14}, \min _{1 \leq i \leq m}\left(w\left(\bar{c}_{j}^{14}\right)\right)=\left[W^{14}\right.$ $\left.\min _{1 \leq i \leq m}\left(w\left(\bar{c}_{j}^{14}\right)\right), W^{14}+\min _{1 \leq i \leq m}\left(w\left(\bar{c}_{j}^{14}\right)\right)\right] \quad$ or $\operatorname{Min} \bar{W}^{14}\left(\bar{y}^{14}\right) \approx \bar{y}^{14} \bar{b}^{14}=\left[W^{* 1}, W^{* 4}\right]$.

Step 4 (Ramesh and Ganesan's method, see [21]): solve the primal and dual (LPIn23) via (LPMi23).

Apply the simplex method to (LPMi23) to determine the primal variables (LPMi23): $x^{23}=\left(\begin{array}{c}x_{1}^{23} \\ x_{2}^{23} \\ \cdot \\ \cdot \\ x_{n+m}^{23}\end{array}\right)$. Assume that $T^{23}$ is optimal, then the current basis is $x_{B}^{23}=\left(\begin{array}{c}x_{J_{1}}^{23}=b_{J_{1}}^{23} \\ x_{J_{2}}^{23}=b_{J_{2}}^{23} \\ \cdot \\ \cdot \\ x_{J_{m}}^{23}=b_{J_{m}}^{23}\end{array}\right)$. Moreover, the current nonbasic variables is $x_{N}^{23}=\left\{x_{d}^{23}\right\}$, and the corresponding solution is $x_{d}^{23}=0$. Hence, the optimal solution to the problem (LPIn23) can be written as $\bar{x}_{J_{k}}^{23} \approx x_{J_{k}}^{23}, \min _{1 \leq i \leq m}(w$ $\left.\left(\bar{b}_{i}^{23}\right)\right)=\left[x_{J_{k}}^{23}-\min _{1 \leq i \leq m}\left(w\left(\bar{b}_{i}^{23}\right)\right), x_{J_{k}}^{23}+\min _{1 \leq i \leq m} \quad(w\right.$ $\left.\left.\left(\bar{b}_{i}^{23}\right)\right)\right]$ for $1 \leq k \leq m$ with the associated value of the objective function: $\bar{Z}^{23} \approx Z^{23}, \min _{1 \leq i \leq m}\left(w\left(\bar{b}_{i}^{23}\right)\right)=\left[Z^{23}\right.$ $\left.-\min _{1 \leq i \leq m}\left(w\left(\bar{b}_{i}^{23}\right)\right), Z^{23}+\min _{1 \leq i \leq m}\left(w\left(\bar{b}_{i}^{23}\right)\right)\right]$ or $\bar{Z}^{23}$ $\left(\bar{x}^{23}\right) \approx \bar{c}^{23} \bar{x}^{23}=\left[Z^{* 2}, Z^{* 3}\right]$.

Then, the corresponding dual variables $(D)$ is given by $\bar{y}^{23} \approx\left(\bar{y}_{1}^{23} \bar{y}_{2}^{23} \ldots \bar{y}_{n+m}^{23}\right)$ in $\bar{T}^{23}$. We have $\bar{y}_{i}^{23} \approx\left|\Delta_{n+i}^{23}\right|$, $\min _{1 \leq i \leq m}\left(w\left(\bar{c}_{j}^{23}\right)\right)=\left[\left|\Delta_{n+i}^{23}\right|-\min _{1 \leq i \leq m}\left(w\left(\bar{c}_{j}^{23}\right)\right),\left|\Delta_{n+i}^{23}\right|\right.$ 
$\left.+\min _{1 \leq i \leq m}\left(w\left(\bar{c}_{j}^{23}\right)\right)\right] \quad$ and $\quad \bar{y}_{m+j}^{23} \approx\left|\Delta_{j}^{23}\right|, \quad \min _{1 \leq i \leq m}$ $\left(w\left(\bar{c}_{j}^{23}\right)\right)=\left[\left|\Delta_{j}^{23}\right|-\min _{1 \leq i \leq m}\left(w\left(\bar{c}_{j}^{23}\right)\right),\left|\Delta_{j}^{23}\right|+\min _{1 \leq i \leq m}\right.$ $\left.\left(w\left(\bar{c}_{j}^{23}\right)\right)\right]$ with the associated value of the objective function Min: $\bar{W}^{23} \approx W^{23}, \min _{1 \leq i \leq m}\left(w\left(\bar{c}_{j}^{23}\right)\right)=\left[W^{23}-\right.$ $\left.\min _{1 \leq i \leq m}\left(w\left(\bar{c}_{j}^{23}\right)\right), W^{23}+\min _{1 \leq i \leq m}\left(w\left(\bar{c}_{j}^{23}\right)\right)\right] \quad$ or $\bar{W}^{23}\left(\bar{y}^{23}\right) \approx \bar{y}^{23} \bar{b}^{23}=\left[W^{* 2}, W^{* 3}\right]$.

Step 5: fuzzy optimal solution of linear programming problems involving trapezoidal fuzzy numbers (LPTra):

(i) Primal optimal solution: $\tilde{x}_{j}^{*} \approx\left(\bar{x}_{j}^{14} \mid \bar{x}_{j}^{23}\right)=\left(\left[x_{j}^{* 1}\right.\right.$, $\left.\left.x_{j}^{* 4}\right] \mid\left[x_{j}^{* 2}, x_{j}^{* 3}\right]\right)=\left(x_{j}^{* 1}, x_{j}^{* 2}, x_{j}^{* 3}, x_{j}^{* 4}\right), \quad j=1$, $\ldots, n+m$, with the associated value of the objective function $\operatorname{Max} \widetilde{Z}^{*} \approx\left(Z^{* 1}, Z^{* 2}, Z^{* 3}, Z^{* 4}\right)=$ $\left(\bar{Z}^{14} \mid \bar{Z}^{23}\right)$.

(ii) Then, the corresponding dual optimal solution problem is given by $\tilde{y}_{i}^{*} \approx\left(\bar{y}_{i}^{14} \mid \bar{y}_{i}^{23}\right)=$ $\left(\left[y_{i}^{* 1}, y_{i}^{* 4}\right] \mid\left[y_{i}^{* 2}, y_{i}^{* 3}\right]\right)=\left(y_{i}^{* 1}, y_{i}^{* 2}, y_{i}^{* 3}, y_{i}^{* 4}\right)$, $i=1, \ldots, n+m$ with the associated value of the objective function $\operatorname{Min} \widetilde{W}^{*} \approx\left(W^{* 1}, W^{* 2}, W^{* 3}\right.$, $\left.W^{* 4}\right)=\left(\bar{W}^{14} \mid \bar{W}^{23}\right)$

(iii) Comparisons using ranking function $\mathscr{R}$ $[12,24,25]$

$\mathscr{R}\left(\operatorname{Max} \widetilde{Z}^{*}\right)=(1 / 4)\left(Z^{* 1}+Z^{* 2}+Z^{* 3}+Z^{* 4}\right)$ and $\mathscr{R}\left(\operatorname{Min} \widetilde{W}^{*}\right)=1 / 4\left(W^{* 1}+W^{* 2}+W^{* 3}+W^{* 4}\right)$.

NB: let $\widetilde{a}=\left(a^{1}, a^{2}, a^{3}, a^{4}\right)$ and $\widetilde{b}=\left(b^{1}, b^{2}, b^{3}, b^{4}\right)$; then, $\tilde{a} \leqslant \widetilde{b}$ iff $\mathscr{R}(\widetilde{a}) \leq \mathscr{R}(\tilde{b}), \tilde{a} \geqslant \tilde{b}$ iff $\mathscr{R}(\widetilde{a}) \geq \mathscr{R}(\widetilde{b})$, and $\tilde{a} \approx \tilde{b}$ iff $\mathscr{R}(\tilde{a})=\mathscr{R}(\tilde{b})$.

\section{Advantages of the Proposed Method}

In this section, the advantages of the proposed method over the existing methods for solving LPTras are discussed:

(1) The proposed approach can be applied for solving fully fuzzy linear programming problems where all the parameters are represented as fuzzy numbers.

(2) The ranking functions of the optimal solutions are nonnegative real numbers, i.e., the midpoint values $m\left(x_{j}^{23}\right)=\left(x_{j}^{3}+x_{j}^{2} / 2\right)$ are no negative.

(3) In contrast to the existing method [12, 25], the proposed method provides fuzzy optimal solutions that indicate possible outcomes with a certain degree of membership to the decision maker. This is especially useful for strategic decisions in cases more uncertainty exists.
(4) The proposed method obeys the strong duality theorem and complementary slackness theorem [21] (i.e., Ramesh and Ganesan [21] have proved the weak and strong duality theorems. Complementary slackness theorem is also proved for the LPIns).

(5) The main advantage of the proposed method is that utilizing problems LPIn14 and LPIn23 for solving LPTra is highly economical compared with problem LPTra from a computational viewpoint, regarding the number of constraints and variables. There is a direct relationship between the computational complexity of LPMi14 and LPMi23 problems and the number of their constraints and variables. Because the memory size needed for maintaining the basis (or its inverse) in the simplex algorithm is given by the square of the number of constraints, reducing the number of constraints in LPMi14 and LPMi23 models is crucial for increasing the computational efficiency.

(6) In contrast to existing methods $[12,25]$, the proposed approach does not utilize fuzzy ranking functions for modelling the objective and constraint functions.

(7) The proposed method is easy to apply for finding the fuzzy optimal solution of LPTra in real-world applications compared with the existing methods.

(8) It is highlighted in $[12,25]$ that the computational efforts required to solve an LPTra problem can be reduced if trapezoidal fuzzy numbers are used to convey the subjective evaluations of decision makers. Using such fuzzy numbers allows us to compare the proposed method with most of the existing approaches in the literature. Because the proposed scheme considers the trapezoidal fuzzy numbers as intervals, the approximated multiplication does not impact the results, i.e., exact formulas yield the same results as the presented approach.

\section{Numerical Examples}

Numerical examples are provided to illustrate the theory developed in this paper.

Example 1. Step 1: consider the primal $(P)$ and dual (LPTra).

Primal $(P)$ :

$$
\begin{aligned}
& \operatorname{Min} \widetilde{Z}=(1,4,8,11) \widetilde{x}_{1}+(2,4,6,8) \widetilde{x}_{2}(1,2,4,5) \widetilde{x}_{3} \\
& (1,2,4,5) \tilde{x}_{1}+(0,2,6,8) \tilde{x}_{2}+(1,2,2,3) \tilde{x}_{3} \geqslant(3,6,10,13) \\
& \text { subject to the constriants } \quad(0,2,6,8) \widetilde{x}_{1}+(1,2,2,3) \widetilde{x}_{2}+(1,1,1,1) \widetilde{x}_{3} \geqslant(2,4,6,8) \\
& (1,2,2,3) \tilde{x}_{1}+(1,1,1,1) \tilde{x}_{2}+(1,2,4,5) \tilde{x}_{3} \geqslant(1,2,6,7),
\end{aligned}
$$




$$
\begin{array}{ll}
\operatorname{Max} \widetilde{W}=(3,6,10,13) \tilde{y}_{1}+ & (2,4,6,8) \tilde{y}_{2}+(1,2,6,7) \tilde{y}_{3} \\
& (1,2,4,5) \tilde{y}_{1}+(0,2,6,8) \tilde{y}_{2}+(1,2,2,3) \widetilde{y}_{3} \preccurlyeq(1,4,8,11) \\
\text { subject to the constraints } \quad(0,2,6,8) \tilde{y}_{1}+(1,2,2,3) \tilde{y}_{2}+(1,1,1,1) \tilde{y}_{3} \preccurlyeq(2,4,6,8) \\
\\
(1,2,2,3) \tilde{y}_{1}+(1,1,1,1) \tilde{y}_{2}+(1,2,4,5) \tilde{y}_{3} \preccurlyeq(1,2,4,5),
\end{array}
$$

where $\tilde{y}_{1}, \tilde{y}_{2}$, and $\tilde{y}_{3}$ are trapezoidal fuzzy numbers.

Step 2: Identify (LPIn14) and (LPIn23):

$$
\begin{aligned}
& \begin{array}{ll}
\operatorname{Min} & \bar{Z}^{14}\left(-x_{1}^{14}, x_{2}^{14}, x_{3}^{14}\right) \approx[1,11] x_{1}^{14}+[2,8] x_{2}^{14}+[1,5] x_{3}^{14} \\
& {[1,5] x_{1}^{14}+[0,8] x_{2}^{14}+[1,3] x_{3}^{14} \pm[3,13]=\frac{13+3}{2}, \frac{13-3}{2}=8,5}
\end{array} \\
& \text { Subject to the contraints } \quad[0,8] x_{1}^{14}+[1,3] x_{2}^{14}+[1,1] x_{3}^{14} \pm[2,8]=5,3 \\
& {[1,3] x_{1}^{14}+[1,1] x_{2}^{14}+[1,5] x_{3}^{14} \pm[1,7]=4,3} \\
& \int \begin{array}{ll}
\text { Min } & \overline{\mathrm{Z}}^{23}\left(\mathrm{x}_{1}^{23}, \mathrm{x}_{2}^{23}, \mathrm{x}_{3}^{23}\right)[4,8] \mathrm{x}_{1}^{23}+[4,6] \mathrm{x}_{2}^{23}+[2,4] \mathrm{x}_{3}^{23} \\
& {[2,4] \mathrm{x}_{1}^{23}+[2,6] \mathrm{x}_{2}^{23}+[2,2] \mathrm{x}_{3}^{23} \pm[6,10]=\frac{10+6}{2}, \frac{10-6}{2}=8,2}
\end{array} \\
& \begin{array}{l}
\overline{\mathrm{Z}}^{23}\left(\mathrm{x}_{1}^{23}, \mathrm{x}_{2}^{23}, \mathrm{x}_{3}^{23}\right)[4,8] \mathrm{x}_{1}^{23}+[4,6] \mathrm{x}_{2}^{23}+[2,4] \mathrm{x}_{3}^{23} \\
{[2,4] \mathrm{x}_{1}^{23}+[2,6] \mathrm{x}_{2}^{23}+[2,2] \mathrm{x}_{3}^{23} \pm[6,10]=\frac{10+6}{2}, \frac{10-6}{2}=8,2}
\end{array} \\
& \left\{\begin{array}{l}
\text { Min } \\
\text { Subject to the contraint }
\end{array}\right. \\
& {[2,6] \mathrm{x}_{1}^{23}+[2,2] \mathrm{x}_{2}^{23}+[1,1] \mathrm{x}_{3}^{23} \pm[4,6]=5,1} \\
& {[2,2] \mathrm{x}_{1}^{23}+[1,1] \mathrm{x}_{2}^{14}+[2,4] \mathrm{x}_{3}^{23} \pm[2,6]=4,2 .}
\end{aligned}
$$

Then, the corresponding dual problem is given by

$$
\begin{aligned}
& \begin{cases}\operatorname{Max} & \tilde{W}^{14} \approx[3,13] y_{1}^{14}+[2,8] y_{2}^{14}+[1,7] y_{3}^{14} \\
& {[1,5] y_{1}^{14}+[0,8] y_{2}^{14}+[1,3] y_{3}^{14} \leqslant[1,11]} \\
\text { Subject to the contraints } & {[0,8] y_{1}^{14}+[1,3] y_{2}^{14}+[1,1] y_{3}^{14} \leqslant[2,8]} \\
& {[1,3] y_{1}^{14}+[1,1] y_{2}^{14}+[1,5] y_{3}^{14} \leqslant[1,5],} \\
& \widetilde{W}^{23} \approx[6,10] y_{1}^{23}+[4,6] y_{2}^{23}+[2,6] y_{3}^{23}\end{cases} \\
& \begin{cases}\text { Max } & {[2,4] y_{1}^{23}+[2,6] y_{2}^{23}+[2,2] y_{3}^{23} \leqslant[4,8]} \\
\text { Subject to the contraints } & {[2,6] y_{1}^{23}+[2,2] y_{2}^{23}+[1,1] y_{3}^{23} \leqslant[4,6]} \\
& {[2,2] y_{1}^{23}+[1,1] y_{2}^{23}+[2,4] y_{3}^{23} \leqslant[2,4] .}\end{cases}
\end{aligned}
$$


Step 3 (Ramesh and Ganesan's method, see [21]): solve the primal and dual (LPIn14) via (LPMi14).

Apply the simplex method to (LPMi14): Min $Z^{14}\left(x^{14}\right)=6 x_{1}^{14}+5 x_{2}^{14}+3 x_{3}^{14}$ subject to $3 x_{1}^{14}+$ $4 x_{2}^{14}+2 x_{3}^{14} \geq 8, \quad 4 x_{1}^{14}+2 x_{2}^{14}+x_{3}^{14} \geq 5, \quad 2 x_{1}^{14}+x_{2}^{14}+$ $3 x_{3}^{14} \geq 4$. (Table 1)

(1) Optimal solution primal to LPIn14:

(i) Optimal solution $x^{14}=\left(x_{1}^{14}, x_{2}^{14}, \ldots, x_{6}^{14}\right)$ to LPMi14: $x_{1}^{14}=(2 / 5), x_{2}^{14}=(7 / 5), x_{3}^{14}=(3 / 5), x_{4}^{14}=0$, $x_{5}^{14}=0$, and $x_{6}^{14}=0$ with Min $Z^{14}=(56 / 5)$.

(ii) Optimal solution $\bar{x}^{*} \approx\left(\bar{x}_{1}^{*}, \bar{x}_{2}^{*}, \ldots, \bar{x}_{6}^{*}\right)$ to LPIn14: $\bar{x}_{J_{k}}^{14} \approx x_{J_{k}}^{14}, \max _{1 \leq i \leq m}\left(w\left(b_{i}^{14}\right)\right)=\left[x_{J_{k}}^{14}-\right.$ $\left.\max _{1 \leq i \leq m}\left(w\left(\bar{b}_{i}^{14}\right)\right), x_{J_{k}}^{14}+\max _{1 \leq i \leq m}\left(w\left(\bar{b}_{i}^{14}\right)\right)\right]$.

We have $\max _{1 \leq i \leq m}\left(w\left(\bar{b}_{i}^{14}\right)\right)=\max _{1 \leq i \leq m}(5,3,3)$ $=5$. We get $\bar{x}_{1}^{14} \approx(2 / 5), 5=[(-23 / 5),(27 / 5)]$, $\bar{x}_{2}^{14} \approx(7 / 5), 5=[(-18 / 5),(32 / 5)], \quad \bar{x}_{3}^{14} \approx(3 / 5)$, $5=[(-22 / 5),(28 / 5)], \quad \bar{x}_{4}^{14} \approx \overline{0}, \quad \bar{x}_{5}^{14} \approx \overline{0}, \quad$ and $\bar{x}_{6}^{14} \approx \overline{0}$.

(iii) Value of the objective function:
$\operatorname{Min} \bar{Z}^{14} \approx Z^{14}, \max _{1 \leq i \leq m} \quad\left(w\left(\bar{b}_{i}^{14}\right)\right)=\left[Z^{14}-\right.$ $\left.\max _{1 \leq i \leq m}\left(w\left(\bar{b}_{i}^{14}\right)\right), Z^{14}+\max _{1 \leq i \leq m}\left(w\left(\bar{b}_{i}^{14}\right)\right)\right]$ or $\operatorname{Min} \bar{Z}^{14}\left(\bar{x}^{14}\right) \approx \bar{c}^{14} \bar{x}^{14}=\left[Z^{* 1}, Z^{* 4}\right]$. We get Min $\bar{Z}^{14}\left(\bar{x}^{14}\right) \approx(56 / 5), 5=[(31 / 5),(81 / 5)]$.

(2) Then, the corresponding dual problem $(D)$ LPIn 14 is given by

(i) Optimal solution dual $y^{14}=\left(y_{1}^{14} \ldots y_{6}^{14}\right)$ to LPMi14 with $y_{i}^{* 14}=\left|\Delta_{n+i}^{14}\right|$ and $y_{m+j}^{* 14}=\left|\Delta_{j}^{14}\right|$. We get $y_{1}^{14}=(4 / 5), \quad y_{2}^{14}=(4 / 5), \quad y_{3}^{14}=(1 / 5)$, $y_{4}^{14}=0, \quad y_{5}^{14}=0$, and $y_{6}^{14}=0$ with $\operatorname{Max}$ $W^{14}=(56 / 5)$.

(ii) Optimal solution dual $\bar{y}^{14} \approx\left(\bar{y}_{1}^{14} \bar{y}_{2}^{14} \ldots \bar{y}_{n+m}^{14}\right)$ to LPIn14. We have

$\bar{y}_{i}^{14} \approx y_{i}^{* 14}, \quad \max _{1 \leq i \leq m}\left(w\left(\bar{c}_{j}^{14}\right)\right)=\left[y_{i}^{* 14}-\right.$ $\left.\max _{1 \leq i \leq m}\left(w\left(\bar{c}_{j}^{14}\right)\right), y_{i}^{* 14}+\max _{1 \leq i \leq m}\left(w\left(\bar{c}_{j}^{14}\right)\right)\right]$ and $\bar{y}_{m+j}^{14} \approx y_{m+j}^{* 14}, \max _{1 \leq i \leq m}\left(w\left(\bar{c}_{j}^{4}\right)\right)=\left[y_{m+j}^{* 14}-\right.$ $\left.\max _{1 \leq i \leq m}\left(w\left(\bar{c}_{j}^{14}\right)\right), y_{m+j}^{* 14}+\max _{1 \leq i \leq m}\left(w\left(\bar{c}_{j}^{14}\right)\right)\right]$.

We have $\max _{1 \leq i \leq m}\left(w\left(\bar{c}_{j}^{14}\right)\right)=\max _{1 \leq i \leq m}(5,3$, $3)=5$. We get $\bar{y}_{1}^{14} \approx(4 / 5), \quad 5=[(-21 / 5)$, $(29 / 5)], \quad \bar{y}_{2}^{14} \approx(4 / 5), 5=[(-21 / 5),(29 / 5)]$, $\bar{y}_{3}^{14} \approx(1 / 5), 5=[(-24 / 5),(26 / 5)], \quad \bar{y}_{4}^{14} \approx \overline{0}$, $\bar{y}_{5}^{14} \approx \overline{0}$, and $\bar{y}_{6}^{14} \approx \overline{0}$.

(iii) Value of the objective function:

$\operatorname{Max} \bar{W}^{14} \approx W^{14}, \quad \max _{1 \leq i \leq m}\left(w\left(\bar{c}_{j}^{14}\right)\right)=\left[W^{14}-\right.$ $\left.\max _{1 \leq i \leq m}\left(w\left(\bar{c}_{j}^{14}\right)\right), W^{14}+\max _{1 \leq i \leq m}\left(w\left(\bar{c}_{j}^{14}\right)\right)\right]$ or $\operatorname{Max} \bar{W}^{14}\left(\bar{y}^{14}\right) \approx \bar{y}^{14} \bar{b}^{14}=\left[W^{* 1}, W^{* 4}\right]$. We get $\operatorname{Max} \bar{W}^{14} \approx(56 / 5), 5=[(31 / 5),(81 / 5)]$.

Step 4 (Ramesh and Ganesan's method, see [21]): Solve the primal and dual (LPIn23) via (LPMi23).
Apply the simplex method to (LPMi23): Min $Z^{23}\left(x^{23}\right)=6 x_{1}^{23}+5 x_{2}^{23}+3 x_{3}^{23}$ subject to $3 x_{1}^{23}+4 x_{2}^{23}$ $+2 x_{3}^{23} \geq 8,4 x_{1}^{23}+2 x_{2}^{23}+x_{3}^{23} \geq 5,2 x_{1}^{23}+x_{2}^{23}+3 x_{3}^{23} \geq$ $4, x_{1}^{23} \leq(22 / 5), x_{2}^{23} \leq(27 / 5), x_{3}^{23} \leq(23 / 5)$.

(1) Optimal solution primal to LPIn23:

(i) Optimal solution $x^{23}=\left(x_{1}^{23}, x_{2}^{23}, \ldots, x_{6}^{23}\right)$ to LPMi23: $x_{1}^{23}=(2 / 5), x_{2}^{23}=(7 / 5), x_{3}^{23}=(3 / 5)$, $x_{4}^{23}=0, \quad x_{5}^{23}=0, \quad$ and $\quad x_{6}^{23}=0 \quad$ with $\operatorname{Min} Z^{23}=(56 / 5)$.

(ii) Optimal solution $\bar{x}^{*} \approx\left(\bar{x}_{1}^{*}, \bar{x}_{2}^{*}, \ldots, \bar{x}_{5}^{*}\right)$ to LPMi23: $\bar{x}_{J_{k}}^{23} \approx x_{J_{k}}^{23}, \min _{1 \leq i \leq m}\left(w\left(\bar{b}_{i}^{23}\right)\right)=\left[x_{J_{k}}^{23}-\min _{1 \leq i \leq m}\right.$ $\left.\left(w\left(\bar{b}_{i}^{23}\right)\right), x_{J_{k}}^{23}+\min _{1 \leq i \leq m}\left(w\left(\bar{b}_{i}^{23}\right)\right)\right]$.

We havemin $\operatorname{lism}_{1 \leq i \leq m}\left(w\left(\bar{b}_{i}^{23}\right)\right)=\min _{1 \leq i \leq m}(2,1$, $2)=1$. We get $\bar{x}_{1}^{23} \approx(2 / 5), 1=[(-3 / 5),(7 / 5)]$, $\bar{x}_{2}^{23} \approx(7 / 5), 1=[(2 / 5),(12 / 5)], \quad \bar{x}_{3}^{23} \approx(3 / 5), 1$ $=[(-2 / 5),(8 / 5)], \bar{x}_{4}^{23} \approx \overline{0}, \bar{x}_{5}^{23} \approx \overline{0}$, and $\bar{x}_{6}^{23} \approx \overline{0}$.

(iii) Value of the objective function:

$\operatorname{Min} \bar{Z}^{23} \approx Z^{23}, \quad \min _{1 \leq i \leq m}\left(w\left(\bar{b}_{i}^{23}\right)\right)=\left[Z^{23}-\right.$ $\left.\min _{1 \leq i \leq m}\left(w\left(\bar{b}_{i}^{23}\right)\right), Z^{23}+\min _{1 \leq i \leq m}\left(w\left(\bar{b}_{i}^{23}\right)\right)\right]$ or $\operatorname{Min} \bar{Z}^{23}\left(\bar{x}^{23}\right) \approx \bar{c}^{23} \bar{x}^{23}=\left[Z^{* 2}, Z^{* 3}\right]$. We get $\operatorname{Min} \bar{Z}^{23}\left(\bar{x}^{23}\right) \approx(56 / 5), 1=[(51 / 5),(61 / 5)]$.

(2) Then, the corresponding dual problem (D) LPIn23 is given by

(i) Optimal solution dual $y^{23}=\left(y_{1}^{23} \ldots y_{6}^{23}\right)$ to LPMi23 with $y_{i}^{* 23}=\left|\Delta_{n+i}^{23}\right|$ and $y_{m+j}^{* 23}=\left|\Delta_{j}^{23}\right|$. We get $y_{1}^{23}=(4 / 5), y_{2}^{23}=(4 / 5), y_{3}^{23}=(1 / 5)$, $y_{4}^{23}=0, \quad y_{5}^{23}=0, \quad$ and $\quad y_{6}^{23}=0 \quad$ with $\operatorname{Max} W^{23}=(56 / 5)$.

(ii) Optimal solution dual $\bar{y}^{23} \approx\left(\bar{y}_{1}^{23} \bar{y}_{2}^{23} \ldots \bar{y}_{n+m}^{23}\right)$ to LPIn23. We have $\bar{y}_{i}^{23} \approx y_{i}^{* 23}, \min _{1 \leq i \leq m}\left(w\left(\bar{c}_{j}^{23}\right)\right)=\left[y_{i}^{* 14}-\min _{1 \leq i \leq m}\right.$ $\left.\left(w\left(\bar{c}_{j}^{23}\right)\right), y_{i}^{* 23}+\min _{1 \leq i \leq m}\left(w\left(\bar{c}_{j}^{23}\right)\right)\right]$ and $\bar{y}_{m+j}^{23} \approx$ $y_{m+j}^{* 23}, \min _{1 \leq i \leq m}\left(w\left(\bar{c}_{j}^{23}\right)\right)=\left[y_{m+j}^{* 23}-\min _{1 \leq i \leq m} \quad(w\right.$ $\left.\left.\left(\bar{c}_{j}^{23}\right)\right), y_{m+j}^{* 23}+\min _{1 \leq i \leq m}\left(w\left(\bar{c}_{j}^{23}\right)\right)\right]$. We have $\min _{1 \leq i \leq m}\left(w\left(\bar{c}_{j}^{23}\right)\right) \min _{1 \leq i \leq m}(2,1,1)=1$. We get $\bar{y}_{1}^{23} \approx(4 / 5), 1=[(-1 / 5),(9 / 5)], \quad \bar{y}_{2}^{23} \approx(4 / 5), 1$ $=[(-1 / 5),(9 / 5)], \quad \bar{y}_{3}^{23} \approx(1 / 5), 1=[(-4 / 5),(6 /$ 5)], $\bar{y}_{4}^{23} \approx \overline{0}, \bar{y}_{5}^{23} \approx \overline{0}$, and $\bar{y}_{6}^{23} \approx \overline{0}$.

(iii) Value of the objective function: $\operatorname{Max} \bar{W}^{23} \approx W^{23}, \min _{1 \leq i \leq m}\left(w\left(\bar{c}_{j}^{23}\right)\right) \quad=\left[W^{23}-\right.$ $\left.\min _{1 \leq i \leq m}\left(w\left(\bar{c}_{j}^{23}\right)\right), W^{23}+\min _{1 \leq i \leq m}\left(w\left(\bar{c}_{j}^{23}\right)\right)\right]$ or $\operatorname{Max} \bar{W}^{23}\left(\bar{y}^{23}\right) \approx \bar{y}^{23} \bar{b}^{23}=\left[W^{* 2}, W^{* 3}\right]$. We get $\operatorname{Max} \bar{W}^{23} \approx(56 / 5), 1=[(51 / 5),(61 / 5)]$.

Step 5: fuzzy optimal solutions and comparisons using ranking function.

(i) Primal optimal solution: 
TABLE 1: The optimal simplex table.

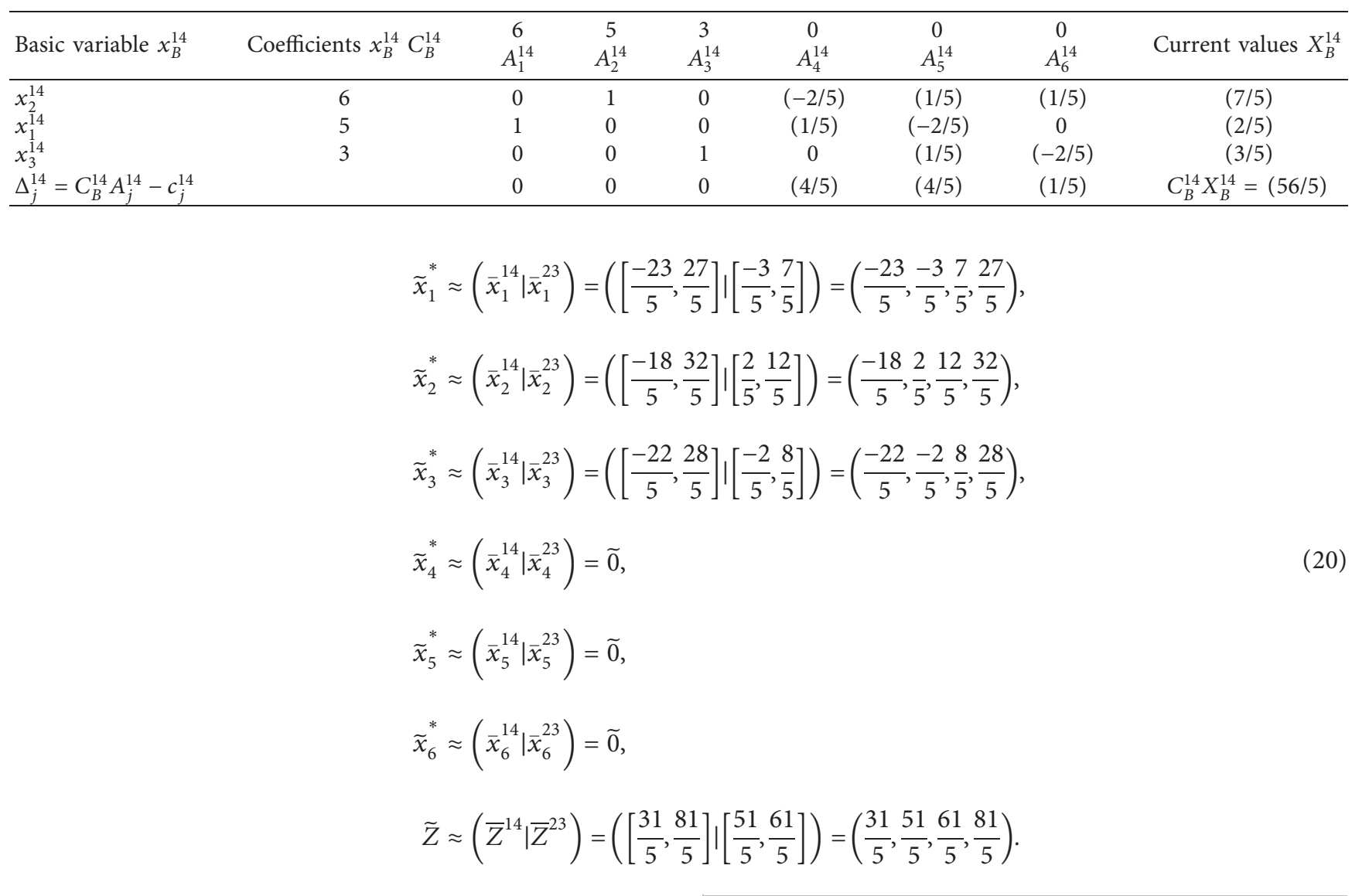

(ii) Dual optimal solution:

$$
\begin{aligned}
\tilde{y}_{1}^{*} & \left.\approx\left(\bar{y}_{1}^{14} \mid \bar{y}_{1}^{23}\right)=\left(\left[\frac{-21}{5}, \frac{29}{5}\right]\right\rfloor\left[\frac{-1}{5}, \frac{9}{5}\right]\right)=\left(\frac{-21}{5}, \frac{-1}{5}, \frac{9}{5}, \frac{29}{5}\right), \\
\tilde{y}_{2}^{*} & \left.\approx\left(\mid \bar{y}_{2}^{23}\right)=\left(\left[\frac{-21}{5}, \frac{29}{5}\right]\right\rfloor\left[\frac{-1}{5}, \frac{9}{5}\right]\right)=\left(\frac{-21}{5}, \frac{-1}{5}, \frac{9}{5}, \frac{29}{5}\right), \\
\tilde{y}_{3}^{*} & \left.\approx\left(\bar{y}_{3}^{14}|| \bar{y}_{3}^{23}\right)=\left(\left[\frac{-24}{5}, \frac{26}{5}\right]\right\rfloor\left[\frac{-4}{5}, \frac{6}{5}\right]\right)=\left(\frac{-24}{5}, \frac{-4}{5}, \frac{6}{5}, \frac{26}{5}\right), \\
\tilde{y}_{4}^{*} & \approx\left(\bar{y}_{4}^{14} \mid y_{4}^{23}\right)=\widetilde{0}, \\
\tilde{y}_{5}^{*} & \approx\left(\bar{y}_{5}^{14} \mid \bar{y}_{5}^{23}\right)=\widetilde{0}, \\
\tilde{y}_{6}^{*} & \approx\left(\bar{y}_{6}^{14} \mid \bar{y}_{6}^{23}\right)=\widetilde{0}, \\
\max \widetilde{W} & \approx\left(\bar{W}^{14} \mid \bar{W}^{23}\right)=\left(\left[\frac{31}{5}, \frac{81}{5}\right] \mid\left[\frac{51}{5}, \frac{61}{5}\right]\right)=\left(\frac{31}{5}, \frac{51}{5}, \frac{61}{5}, \frac{81}{5}\right) .
\end{aligned}
$$


(iii) Comparisons using ranking function Example 2. Step 1: consider the primal (P) and dual $[12,24,25]$ :

$$
\mathscr{R}(\operatorname{Min} \widetilde{Z})=\mathscr{R}(\operatorname{Max} \widetilde{W})=\frac{214}{20} .
$$
(LPTra).

Primal $(P)$ :

$$
\begin{aligned}
& \operatorname{Max} \widetilde{Z}=(11,13,15,17) \widetilde{x}_{1}+(9,12,14,17) \widetilde{x}_{2}+(13,15,17,19) \widetilde{x}_{3} \\
& (1,10,14,23) \widetilde{x}_{1}+(5,10,16,21) \widetilde{x}_{2}+(1,10,14,23) \widetilde{x}_{3} \preccurlyeq(469,475,505,511) \\
& \text { subject to the constraint } \quad(4,13,15,24) \tilde{x}_{1}+(5,10,16,21) \tilde{x}_{3} \preccurlyeq(452,460,480,488) \\
& (1,10,14,23) \widetilde{x}_{1}+(13,15,15,17) \widetilde{x}_{2} \preccurlyeq(460,465,495,500),
\end{aligned}
$$

where $\widetilde{x}_{1}, \widetilde{x}_{2}$, and $\widetilde{x}_{3}$ are trapezoidal fuzzy numbers.

Then, the corresponding dual problem is given by $(D)$ :

$$
\begin{aligned}
& \operatorname{Min} \widetilde{W}=(469,475,505,511) \widetilde{y}_{1}+(452,460,480,488) \widetilde{y}_{2}+(460,465,495,500) \widetilde{y}_{3} \\
& \qquad \begin{array}{cl}
(1,10,14,23) \widetilde{y}_{1}+(4,13,15,24) \tilde{y}_{2}(5,10,16,21) \widetilde{y}_{1}+(13,15,15,17) \\
\text { subject to the constraint } \quad \widetilde{y}_{3} \succcurlyeq(9,12,14,17)+(1,10,14,23) \widetilde{y}_{3} \succcurlyeq(11,13,15,17) \\
& (1,10,14,23) \widetilde{y}_{1}+(5,10,16,21) \tilde{y}_{2} \succcurlyeq(13,15,17,19),
\end{array}
\end{aligned}
$$

where $\tilde{y}_{1}, \tilde{y}_{2}$, and $\tilde{y}_{3}$ are trapezoidal fuzzy numbers.

Step 2: identify (LPIn14) and (LPIn23):

$$
\begin{aligned}
& \operatorname{Max} \\
& \bar{Z}^{14}\left(-x_{1}^{14}, x_{2}^{14}, x_{3}^{14}\right) \approx[11,17] x_{1}^{14}+[9,17] x_{2}^{14}+[13,19] x_{3}^{14} \\
& {[1,23] x_{1}^{14}+[5,21] x_{2}^{14}+\left[1,23\left\lceil x_{3}^{14} \leqslant[469,511]=\frac{511+469}{2}, \frac{511-469}{2}=\langle 490,21\rangle\right.\right.} \\
& \text { (LPIn14) } \\
& {\left[4,24 \sqrt{x_{1}^{14}}+[5,21] x_{3}^{14} \leqslant[452,488]=\langle 470,18\rangle\right.} \\
& {[1,23] x_{1}^{14}+[13,17] x_{2}^{14} \leqslant[460,500]=\langle 480,20\rangle,} \\
& \bar{Z}^{23}\left(x_{1}^{23}, x_{2}^{23}, x_{3}^{23}\right) \approx\left[13,15 \sqrt{x_{1}^{23}}+[12,14] x_{2}^{23}+[15,17] x_{3}^{23}\right. \\
& {[10,14] x_{1}^{23}+[10,16] x_{2}^{23}+[10,14] x_{3}^{23} \leqslant[475,505]=\frac{505+475}{2}, \frac{505-475}{2}=\langle 490,15\rangle} \\
& {[13,15] x_{1}^{23}+[10,16] x_{3}^{23} \leqslant[460,480]=\langle 470,10\rangle} \\
& {[10,14] x_{1}^{23}+[15,15] x_{2}^{14} \leqslant[465,495]=\langle 480,15\rangle .}
\end{aligned}
$$


Then, the corresponding dual problem is given by

$$
\begin{aligned}
& \begin{cases}\text { Min } & \widetilde{W}^{14} \approx[469,511] y_{1}^{14}+[452,488] y_{2}^{14}+[460,500] y_{3}^{14} \\
& {[1,23] y_{1}^{14}+[4,24] y_{2}^{14}+[1,23] y_{3}^{14} \geqslant[11,17]} \\
\text { Subject to the contraints } & {[5,21] y_{1}^{14}+[13,17] y_{3}^{14} \geqslant[9,17]} \\
& {[1,23] y_{1}^{14}+[5,21] y_{2}^{14} \geqslant[13,19],} \\
& \widetilde{W}^{23} \approx[475,505] y_{1}^{23}+[460,480] y_{2}^{23}+[465,495] y_{3}^{23}\end{cases} \\
& \begin{cases}\text { Min } & {[10,14] y_{1}^{23}+[13,15] y_{2}^{23}+[10,14] y_{3}^{23} \geqslant[13,15]} \\
\text { Subject to the contraints } & {[10,16] y_{1}^{23}+[15,15] y_{3}^{23} \geqslant[12,14]} \\
& {[10,14] y_{1}^{23}+[10,16] y_{2}^{23} \geqslant[15,17] .}\end{cases}
\end{aligned}
$$

Step 3 (Ramesh and Ganesan's method, see [21]): solve the primal and dual (LPIn14) via (LPMi14).

Apply the simplex method to (LPMi14) $\operatorname{Max}^{14}\left(x^{14}\right)=14 x_{1}^{14}+13 x_{2}^{14}+16 x_{3}^{14}$ subject to $12 x_{1}^{14}+13 x_{2}^{14}+12 x_{3}^{14} \leq 490, \quad 14 x_{1}^{14}+13 x_{3}^{14} \leq 470$, $12 x_{1}^{14}+15 x_{2}^{14} \leq 480$ (Table 2).

(1) Optimal solution primal to LPIn14.

(i) Optimal solution $x^{14}=\left(x_{1}^{14}, x_{2}^{14}, \ldots, x_{6}^{14}\right)$ to LPMi14:

$$
x_{1}^{14}=0 \text {, }
$$$$
x_{2}^{14}=\frac{730}{169}
$$$$
x_{3}^{14}=\frac{6110}{169},
$$

$x_{4}^{14}=0$,

$x_{5}^{14}=0$,

$x_{6}^{14}=\frac{70170}{169}$ with $\operatorname{Max} Z^{14}=\frac{107250}{169}$.

(ii) Optimal solution $\bar{x}^{*} \approx\left(\bar{x}_{1}^{*}, \bar{x}_{2}^{*}, \ldots, \bar{x}_{6}^{*}\right)$ to LPIn14:

$$
\begin{aligned}
\bar{x}_{J_{k}}^{14} \approx & x_{J_{k}}^{14}, \max _{1 \leq i \leq m}\left(w\left(\bar{b}_{i}^{14}\right)\right)=\left[x_{J_{k}}^{14}-\max _{1 \leq i \leq m}\left(w\left(\bar{b}_{i}^{14}\right)\right), x_{J_{k}}^{14}\right. \\
& \left.+\max _{1 \leq i \leq m}\left(w\left(\bar{b}_{i}^{14}\right)\right)\right] .
\end{aligned}
$$

We havemax ${ }_{1 \leq i \leq m}\left(w\left(\bar{b}_{i}^{14}\right)\right) \max _{1 \leq i \leq m}(21,18,20)$ $=21$. We get $\bar{x}_{1}^{14} \approx \overline{0}$,

$$
\begin{aligned}
& \bar{x}_{2}^{14} \approx \frac{730}{169}, \\
& 21=\left[\frac{-2819}{169}, \frac{4279}{169}\right], \\
& \bar{x}_{3}^{14} \approx \frac{6110}{169}, \\
& 21=\left[\frac{-2561}{169}, \frac{9659}{169}\right], \\
& \bar{x}_{4}^{14} \approx \overline{0}, \\
& \bar{x}_{5}^{14} \approx \overline{0}, \\
& \bar{x}_{6}^{14} \approx \frac{70170}{169}, \\
& 21=\left[\frac{66621}{169}, \frac{73719}{169}\right] .
\end{aligned}
$$

(iii) Value of the objective function:

$\operatorname{Max}\left\langle\bar{Z}^{14} \approx Z^{14}, \quad \max _{1 \leq i \leq m}\left(w\left(\bar{b}_{i}^{14}\right)\right)\right\rangle=\left[Z^{14}-\right.$ $\left.\max _{1 \leq i \leq m}\left(w\left(\bar{b}_{i}^{14}\right)\right), Z^{14}+\max _{1 \leq i \leq m}\left(w\left(\bar{b}_{i}^{14}\right)\right)\right]$ or $\operatorname{Max} \bar{Z}^{14}\left(\bar{x}^{14}\right) \approx \bar{c}^{14} \bar{x}^{14}=\left[Z^{* 1}, Z^{* 4}\right]$. We get $\operatorname{Max} \quad \bar{Z}^{14}\left(\bar{x}^{14}\right) \approx\langle(107250 / 169), 21\rangle=$ [(103701/169), (110799/169)].

(2) Then, the corresponding dual problem (D) LPIn14 is given by

(i) Optimal solution dual $y^{14}=\left(y_{1}^{14} \ldots y_{6}^{14}\right)$ to LPMi14 with $y_{i}^{* 14}=\left|\Delta_{n+i}^{14}\right|$ and $y_{m+j}^{* 14}=\left|\Delta_{j}^{14}\right|$. We get $y_{1}^{14}=1, \quad y_{2}^{14}=(4 / 13), \quad y_{3}^{14}=0$, $y_{4}^{14}=(30 / 13), \quad y_{5}^{14}=0 \quad$ and $\quad y_{6}^{14}=0 \quad$ with $\operatorname{Min} W^{14}=(107250 / 169)$.

(ii) Optimal solution dual $\bar{y}^{14} \approx\left(\bar{y}_{1}^{14} \bar{y}_{2}^{14} \ldots \bar{y}_{n+m}^{14}\right)$ to LPIn14. We have 
TABLE 2: The optimal simplex table.

\begin{tabular}{lcccccccc}
\hline Basic variable $x_{B}^{14}$ & Coefficients $x_{B}^{14} C_{B}^{14}$ & 14 & 13 & 16 & 0 & 0 & 0 & Current values $X_{B}^{14}$ \\
\hline$x_{2}^{14}$ & & $A_{1}^{14}$ & $A_{2}^{14}$ & $A_{3}^{14}$ & $A_{4}^{14}$ & $A_{5}^{14}$ & $A_{6}^{14}$ & $(730 / 169)$ \\
$x_{3}^{14}$ & 13 & $(-12 / 169)$ & 1 & 0 & $(1 / 13)$ & $(-12 / 169)$ & 0 & $(470 / 13)$ \\
$x_{6}^{14}$ & 16 & $(14 / 13)$ & 0 & 1 & 0 & $(1 / 13)$ & 0 & $(70170 / 169)$ \\
$\Delta_{j}^{14}=C_{B}^{14} A_{j}^{14}-c_{j}^{14}$ & 0 & $(2208 / 169)$ & 0 & 0 & $(-15 / 13)$ & $(180 / 169)$ & 1 & 0 \\
\hline
\end{tabular}

$$
\text { get } \begin{aligned}
\bar{y}_{1}^{14} \approx 1,4=[-3,5], \\
\bar{y}_{2}^{14} \approx \frac{4}{13}, \\
4=\left[\frac{-48}{13}, \frac{56}{13}\right], \\
\bar{y}_{3}^{14} \approx \overline{0}, \\
\bar{y}_{4}^{14} \approx \frac{30}{13}, \\
4=\left[\frac{-22}{13}, \frac{82}{13}\right], \\
\bar{y}_{5}^{14} \approx \overline{0}, \\
\bar{y}_{6}^{14} \approx \overline{0} .
\end{aligned}
$$$$
\bar{y}_{i}^{14} \approx y_{i}^{* 14}, \max _{1 \leq j \leq n}\left(w\left(\bar{c}_{j}^{14}\right)\right)=\left[y_{i}^{* 14}-\max _{1 \leq j \leq n}\right.
$$$$
\left.\left(w\left(\bar{c}_{j}^{14}\right)\right), y_{i}^{* 14}+\max _{1 \leq j \leq n}\left(w\left(\bar{c}_{j}^{14}\right)\right)\right] \text { and } \bar{y}_{m+j}^{14} \approx
$$$$
y_{m+j}^{* 14}, \max _{1 \leq j \leq n}\left(w\left(\bar{c}_{j}^{14}\right)\right)=\left[y_{m+j}^{* 14}-\max _{1 \leq j \leq n}(w\right.
$$$$
\left.\left.\left(\bar{c}_{j}^{14}\right)\right), y_{m+j}^{* 14}+\max _{1 \leq j \leq n}\left(w\left(\bar{c}_{j}^{14}\right)\right)\right] . \quad \text { We }
$$$$
\text { havemax }_{1 \leq j \leq n}\left(w\left(\bar{c}_{j}^{14}\right)\right) \max _{1 \leq j \leq n}(3,4,3)=4 \text {. We }
$$

(iii) Value of the objective function:

$\operatorname{Min} \bar{W}^{14} \approx W^{14}, \max _{1 \leq j \leq n}\left(w\left(\bar{c}_{j}^{14}\right)\right)=\left[W^{14}-\right.$ $\left.\max _{1 \leq j \leq n}\left(w\left(\bar{c}_{j}^{14}\right)\right), W^{14}+\max _{1 \leq j \leq n}\left(w\left(\bar{c}_{j}^{14}\right)\right)\right]$ or $\operatorname{Min} \bar{W}^{14}\left(\bar{y}^{14}\right) \approx \bar{y}^{14} \bar{b}^{14}=\left[W^{* 1}, W^{* 4}\right]$. We get $\operatorname{Min} \bar{W}^{14} \approx(107250 / 169), \quad 4=[(106574 / 169)$, (107926/169)].

Step 4 (Ramesh and Ganesan's method, see [21]): solve the primal and dual (LPIn23) via (LPMi23).

Apply the simplex method to (LPMi23) Max $Z^{23}$ $\left(x^{23}\right)=14 x_{1}^{23}+13 x_{2}^{23}+16 x_{3}^{23}$ subject to $12 x_{1}^{23}+$ $13 x_{2}^{23}+12 x_{3}^{23} \leq 490, \quad 14 x_{1}^{23}+13 x_{3}^{23} \leq 470, \quad 12 x_{1}^{23}+$ $15 x_{2}^{23} \leq 15$ :

$$
\begin{array}{r}
x_{2}^{23} \leq \frac{2589}{169}, \\
\frac{4251}{169} \leq x_{3}^{23} \leq \frac{7969}{169} .
\end{array}
$$

(1) Optimal solution primal to LPIn23.

(i) Optimal solution $x^{23}=\left(x_{1}^{23}, x_{2}^{23}, \ldots, x_{6}^{23}\right)$ to LPMi23: $x_{1}^{23}=0$,

$x_{2}^{23}=\frac{730}{169}$

$x_{3}^{23}=\frac{6110}{169}$

$x_{4}^{23}=0$,

$x_{5}^{23}=0$ and $x_{6}^{23}=\frac{70170}{169}$ With $\operatorname{Max} Z^{23}=\frac{107250}{169}$.

(ii) Optimal solution $\bar{x}^{*} \approx\left(\bar{x}_{1}^{*}, \bar{x}_{2}^{*}, \ldots, \bar{x}_{5}^{*}\right)$ to LPIn23:

$\bar{x}_{J_{k}}^{23} \approx x_{J_{k}}^{23}, \min _{1 \leq i \leq m}\left(w\left(\bar{b}_{i}^{23}\right)\right)=\left[x_{J_{k}}^{23}-\min _{1 \leq i \leq m}\right.$ $\left.\left(w\left(\bar{b}_{i}^{23}\right)\right), x J_{k}^{23}+\min _{1 \leq i \leq m}\left(w\left(\bar{b}_{i}^{23}\right)\right)\right]$. We have $\min _{1 \leq i \leq m}\left(w\left(\bar{b}_{i}^{23}\right)\right) \min _{1 \leq i \leq m}(15,10,15)=10$. We get $\bar{x}_{1}^{23} \approx \overline{0}, \quad \bar{x}_{2}^{23} \approx(730 / 169), 10=[(-960 /$ 169), (2420/169)], $\quad \bar{x}_{3}^{23} \approx(6110 / 169), 10=$ [(4420/169), (7800/169)], $\bar{x}_{4}^{23} \approx \overline{0}, \bar{x}_{5}^{23} \approx \overline{0}$, and $\bar{x}_{6}^{23} \approx(70170 / 169), 10=[(68480 / 169)$,

(71860/169)]

(iii) Value of the objective function:

$\operatorname{Max} \bar{Z}^{23} \approx Z^{23}, \quad \min _{1 \leq i \leq m}\left(w\left(\bar{b}_{i}^{23}\right)\right)=\left[Z^{23}-\right.$ $\left.\min _{1 \leq i \leq m}\left(w\left(\bar{b}_{i}^{23}\right)\right), Z^{23}+\min _{1 \leq i \leq m}\left(w\left(\bar{b}_{i}^{23}\right)\right)\right]$ or $\operatorname{Max} \bar{Z}^{23}\left(\bar{x}^{23}\right) \approx \bar{c}^{23} \bar{x}^{23}=\left[Z^{* 2}, Z^{* 3}\right]$. We get $\operatorname{Max} \bar{Z}^{23}\left(\bar{x}^{23}\right) \approx(107250 / 169), \quad 10=[(105560 /$ 169), (108940/169)].

(2) Then, the corresponding dual problem (D) LPIn23 is given by

(i) Optimal solution dual $y^{23}=\left(y_{1}^{23} \ldots y_{6}^{23}\right)$ to LPMi23 with $y_{i}^{* 23}=\left|\Delta_{n+i}^{23}\right|$ and $y_{m+j}^{* 23}=\left|\Delta_{j}^{23}\right|$. We get $y_{1}^{23}=1, \quad y_{2}^{23}=(4 / 13)$, $y_{3}^{23}=0, \quad y_{4}^{23}=(30 / 13), \quad y_{5}^{23}=0, \quad$ and $y_{6}^{23}=0$ with $W^{23}=(107081 / 169)$.

(ii) Optimal solution dual $\bar{y}^{23} \approx\left(\bar{y}_{1}^{23} \bar{y}_{2}^{23} \ldots \bar{y}_{n+m}^{23}\right)$ to LPIn23. We have $\bar{y}_{i}^{23} \approx y_{i}^{* 23}, \min _{1 \leq j \leq n}\left(w\left(\bar{c}_{j}^{23}\right)\right)=\left[y_{i}^{* 14}-\min \right.$


$\bar{y}_{m+j}^{23} \approx y_{m+j}^{* 23}, \min _{1 \leq j \leq n}\left(w\left(\bar{c}_{j}^{23}\right)\right)=\left[y_{m+j}^{* 23}-\min \right.$ $\left.1 \leq j \leq n \quad\left(w\left(\bar{c}_{j}^{23}\right)\right), y_{m+j}^{* 23}+\min _{1 \leq j \leq n}\left(w\left(\bar{c}_{j}^{23}\right)\right)\right]$. We have $\min _{1 \leq j \leq n}\left(w\left(\bar{c}_{j}^{23}\right)\right) \min _{1 \leq j \leq n}(1,1,1)=1$. We 
get $\bar{y}_{1}^{23} \approx 1,1=[0,2], \bar{y}_{2}^{23} \approx(4 / 13), 1=[(-9 /$ 13), (17/13)], $\bar{y}_{3}^{23} \approx \overline{0}, \bar{y}_{4}^{23} \approx(30 / 13), 1=[(17)$, $(43 / 13)], \bar{y}_{5}^{23} \approx \overline{0}$, and $\bar{y}_{6}^{23} \approx \overline{0}$.

(iii) Value of the objective function:

$\operatorname{Min} \bar{W}^{23} \approx W^{23}, \min _{1 \leq j \leq n}\left(w\left(\bar{c}_{j}^{23}\right)\right)=\left[W^{23}-\right.$ $\left.\min _{1 \leq j \leq n}\left(w\left(\bar{c}_{j}^{23}\right)\right), W^{23}+\min _{1 \leq j \leq n}\left(w\left(\bar{c}_{j}^{23}\right)\right)\right]$ or $\operatorname{Min} \bar{W}^{23}\left(\bar{y}^{23}\right) \approx \bar{y}^{23} \bar{b}^{23}=\left[W^{* 2}, W^{* 3}\right]$. We get
$\operatorname{Min} \bar{W}^{23} \approx(107251 / 169), \quad 1=[(107081 / 169)$, (107419/169)].

Step 5: fuzzy optimal solutions and comparisons using ranking function.

(i) Primal optimal solution:

$$
\begin{aligned}
\tilde{x}_{1}^{*} \approx\left(\bar{x}_{1}^{14} \mid \bar{x}_{1}^{23}\right) & =\widetilde{0} \\
\tilde{x}_{2}^{*} \approx\left(\bar{x}_{2}^{14} \mid \bar{x}_{2}^{23}\right) & =\left(\left[\frac{-2819}{169}, \frac{4279}{169}\right] \mid\left[\frac{-960}{169}, \frac{2420}{169}\right]\right)=\left(\frac{-2819}{169}, \frac{-960}{169}, \frac{2420}{169}, \frac{4279}{169}\right), \\
\tilde{x}_{3}^{*} \approx\left(\bar{x}_{3}^{14} \mid \bar{x}_{3}^{23}\right) & =\left(\left[\frac{2561}{169}, \frac{9659}{169}\right] \mid\left[\frac{4420}{169}, \frac{7800}{169}\right]\right)=\left(\frac{2561}{169}, \frac{4420}{169}, \frac{7800}{169}, \frac{9659}{169}\right), \\
\tilde{x}_{4}^{*} \approx\left(\bar{x}_{4}^{14} \mid \bar{x}_{4}^{23}\right) & =\widetilde{0} \\
\tilde{x}_{5}^{*} \approx\left(\bar{x}_{5}^{14} \mid \bar{x}_{5}^{23}\right) & =\widetilde{0}, \\
\tilde{x}_{6}^{*} \approx\left(\bar{x}_{6}^{14} \mid \bar{x}_{6}^{23}\right) & =\left(\left[\frac{66621}{169}, \frac{73719}{169}\right] \mid\left[\frac{68480}{169}, \frac{71860}{169}\right]\right)=\left(\frac{66621}{169}, \frac{68480}{169}, \frac{71860}{169}, \frac{73719}{169}\right), \\
\operatorname{Max} \widetilde{Z} \approx\left(\bar{Z}^{14} \mid \bar{Z}^{23}\right) & =\left(\left[\frac{103701}{169}, \frac{110799}{169}\right] \mid\left[\frac{105560}{169}, \frac{108940}{169}\right]\right)=\left(\frac{103701}{169}, \frac{105560}{169}, \frac{108940}{169}, \frac{110799}{169}\right) .
\end{aligned}
$$

(ii) Dual optimal solution:

$$
\begin{aligned}
& \tilde{y}_{1}^{*} \approx\left(\bar{y}_{1}^{14} \mid \bar{y}_{1}^{23}\right)=([-3,5] \mid[0,2])=(-3,0,2,5), \\
& \tilde{y}_{2}^{*} \approx\left(\bar{y}_{2}^{14} \mid \bar{y}_{2}^{23}\right)=\left(\left[\frac{-48}{13}, \frac{56}{13}\right] \mid\left[\frac{-9}{13}, \frac{17}{13}\right]\right)=\left(\frac{-48}{13}, \frac{-9}{13}, \frac{17}{13}, \frac{56}{13}\right), \\
& \tilde{y}_{3}^{*} \approx\left(\bar{y}_{3}^{14} \mid \bar{y}_{3}^{23}\right)=\widetilde{0} \\
& \tilde{y}_{4}^{*} \approx\left(\bar{y}_{4}^{14} \mid \bar{y}_{4}^{23}\right)=\left(\left[\frac{-22}{13}, \frac{82}{13}\right] \mid\left[\frac{17}{13}, \frac{43}{13}\right]\right)=\left(\frac{-22}{13}, \frac{17}{13}, \frac{43}{13}, \frac{82}{13}\right), \\
& \tilde{y}_{5}^{*} \approx\left(\bar{y}_{5}^{14} \mid \bar{y}_{5}^{23}\right)=\widetilde{0}, \\
& \tilde{y}_{6}^{*} \approx\left(\bar{y}_{6}^{14} \mid \bar{y}_{6}^{23}\right)=\widetilde{0},
\end{aligned}
$$

$$
\operatorname{Min} \tilde{W} \approx\left(\bar{W}^{14} \mid \bar{W}^{23}\right)=\left(\left[\frac{106574}{169}, \frac{107926}{169}\right] \mid\left[\frac{107081}{169}, \frac{107419}{169}\right]\right)=\left(\frac{106574}{169}, \frac{107081}{169}, \frac{107419}{169}, \frac{107926}{169}\right)
$$

(iii) Comparisons using ranking function $[12,24,25]$ :

$$
\mathscr{R}(\operatorname{Max} \widetilde{Z})=\mathscr{R}(\operatorname{Min} \widetilde{W})=\frac{107250}{169} .
$$

\section{Comparative Study}

To compare the existing methods [12-20, 25] and the methods presented in this paper, the results of semifully fuzzy linear programming (SFFLP) problems and linear programming problems involving trapezoidal fuzzy 
numbers (LPTra), obtained by using the existing methods and the methods presented in this paper, are explained as follows.

(1) The results presented in [12] can be explained as follows:

(i) Nasseri et al. [12] considered a kind of linear programming problems (SFFLP) where

(a)The cost coefficients $\left(\widetilde{c}_{j}\right)$ in objective function are symmetric trapezoidal fuzzy numbers

(b)The right-hand side vectors $\left(\tilde{b}_{i}\right)$ are symmetric trapezoidal fuzzy numbers

(c)The variables $\left(\tilde{x}_{j}\right)$ are symmetric trapezoidal fuzzy numbers

(d)The coefficients $\left(a_{i j}\right)$ are real numbers

(ii) In the LPTra method proposed in this study [12], the coefficients of the objective function and the values of the right-hand side are represented by symmetric trapezoidal fuzzy numbers, while the elements of the coefficient matrix are represented by real numbers. The optimal solution of the LPTra problem is simply found by solving an equivalent crisp LP problem. The LPTra problem is converted into a crisp equivalent LP problem and the crisp LP problem is solved with the standard primal simplex method.

(iii) The generalization of the proposed method [12] to overcome this shortcoming is left to future research in LPTra problems with symmetric trapezoidal fuzzy numbers. Finally, we point out that the LPTra method proposed in this study [12] does not consider fuzzy cost coefficients and a fuzzy constraint matrix. Developing a full fuzzy version of the proposed method and overcoming this limitation is an interesting stream of future research.

(2) The results presented by our computational method can be explained as follows.

(i) In this paper consider a kind of linear programming problems (LPTra) where

(a)The cost coefficients $\left(\widetilde{c}_{j}\right)$ in objective function are trapezoidal fuzzy numbers

(b)The right-hand side vector $\left(\widetilde{b}_{i}\right)$ are trapezoidal fuzzy numbers

(c)The variables $\left(\tilde{x}_{j}\right)$ are trapezoidal fuzzy numbers

(d)The coefficients $\left(a_{i j}\right)$ are symmetric trapezoidal fuzzy numbers

(ii) In the problems, chosen in Examples 1 and 2, all the coefficients are symmetric trapezoidal fuzzy numbers. So, due to the limitations of the existing method [12], none of these problems can be solved by using the existing method [12]. However, in the problem, chosen in Examples 1 and 2, all the coefficients are represented by symmetric trapezoidal fuzzy numbers. So, as discussed in [12], it can be solved by using the existing method [12], but due to the shortcoming of the existing method [12], the obtained results are not exact.

(iii) Our computational method, presented in Section 3, can be used to find the fuzzy optimal solution of fully fuzzy linear programming problems with symmetric trapezoidal fuzzy numbers coefficients. So, all the problems, chosen in Examples 1 and 2, can be solved by using our computational method, presented in Section 3. Also, as discussed in Section 3, the results obtained by using our computational method, presented in Section 3, are exact.

(iv) Finally, we point out that the LPTra computational method proposed in this study considers fuzzy cost coefficients and a fuzzy constraint matrix.

\section{Conclusion}

In contrast to conventional linear programming problems, we investigated imprecise data for the real-life linear programming problems. In the LPTra considered in this study, all the parameters were represented by trapezoidal fuzzy numbers. We proposed a new method for solving an LPTra by converting it into two linear programming problems involving interval numbers. We considered LPMi14 and LPMi23 problems required to solve the LPTra that had at most constraints and variables compared with existing linear programming problems. Using four application examples, we compared the results obtained using the proposed algorithm and those obtained using with the existing methods, demonstrating the reliability and applicability of our algorithm. Because the proposed method is based on the classical linear programming (LPMi14 and LPMi23) simplex algorithm, it is easy to learn and apply for obtaining fuzzy optimal solutions of LPTras pertaining to real-world applications. One of the main advantages of the proposed method is that both the obtained fuzzy optimal solution and the fuzzy optimal value have nonnegative midpoint values $m\left(x_{j}^{23}\right)=\left(x_{j}^{3}+x_{j}^{2} / 2\right)$. The generalization of the proposed method to overcome this shortcoming is left to future research on LPTra problems with nonsymmetrical fuzzy trapezoidal stress matrix coefficients. Finally, we recall that the LPTra method proposed in this study does not take into account the coefficients of the nonsymmetrical fuzzy trapezoidal stress matrices. Developing a complete fuzzy version of the proposed method and overcoming this limitation is an interesting stream of future research and more important on sensitivity analysis.

\section{Data Availability}

No data were used to support this study.

\section{Conflicts of Interest}

The authors declare no conflicts of interest. 


\section{References}

[1] A. Sengupta and T. K. Pal, "On comparing interval numbers," European Journal of Operational Research, vol. 127, no. 1, pp. $28-43,2000$.

[2] A. Sengupta, T. K. Pal, and D. Chakraborty, "Interpretation of inequality constraints involving interval coefficients and a solution to interval linear programming," Fuzzy Sets and Systems, vol. 119, no. 1, pp. 129-138, 2001.

[3] G. R. Bitran, "Linear multiple objective problems with interval coefficients," Management Science, vol. 26, no. 7, pp. 694-706, 1980.

[4] S. Chanas and D. Kuchta, "Multiobjective programming in optimization of interval objective functions-a generalized approach," European Journal of Operational Research, vol. 94, no. 3, pp. 594-598, 1996.

[5] Y. Nakahara, M. Sasaki, and M. Gen, "On the linear programming problems with interval coefficients," Computers \& Industrial Engineering, vol. 23, no. 1-4, pp. 301-304, 1992.

[6] R. E. Steuer, "Algorithms for linear programming problems with interval objective function coefficients," Mathematics of Operations Research, vol. 6, no. 3, pp. 333-348, 1981.

[7] T. Shaocheng, "Interval number and fuzzy number linear programming," Fuzzy Sets and Systems, vol. 66, pp. 301-306, 1994.

[8] K. Ganesan and P. Veeramani, "On arithmetic operations of interval numbers," International Journal of Uncertainty, Fuzziness and Knowledge-Based Systems, vol. 13, no. 6, pp. 619-631, 2005.

[9] K. Ganesan, "On some properties of interval matrices," International Journal of Computational and Mathematical Sciences, vol. 1, no. 2, pp. 92-99, 2007.

[10] G. Ramesh and K. Ganesan, "Interval linear programming with generalized interval arithmetic," International Journal of Scientifice Engineering Research, vol. 2, no. 11, pp. 2229-5518, 2011.

[11] R. E. Bellman and L. A. Zadeh, "Decision making in a fuzzy environment," Management Science, vol. 17, pp. 141-164, 1970.

[12] S. H. Nasseri, E. Ali, and B.-Y. Cao, "Fuzzy linear programming: solution techniques and applications," Studies in Fuzziness and Soft Computing, Vol. 379, Springer International Publishing, Switzerland, 2019.

[13] N. Mahdavi-Amiri and S. H. Nasseri, "Duality results and a dual simplex method for linear programming problems with trapezoidal fuzzy variables," Fuzzy Sets and Systems, vol. 158, no. 17, pp. 1961-1978, 2007.

[14] A. Ebrahimnejad, "Some new results in linear programs with trapezoidal fuzzy numbers: finite convergence of the Ganesan and Veeramani's method and a fuzzy revised simplex method," Applied Mathematical Modelling, vol. 35, no. 9, pp. 4526-4540, 2011.

[15] E. Ali, "A method for solving linear programming with interval-valued trapezoidal fuzzy variables," RAIRO-Operations Research, vol. 52, no. 3, pp. 955-979, 2018.

[16] E. Ali, "An effective computational attempt for solving fully fuzzy linear programming using MOLP problem," Journal of Industrial and Production Engineering, vol. 36, no. 2, 2019.

[17] E. Ali, "A duality approach for solving bounded linear programming problems with fuzzy variables based on ranking functions and its application in bounded transportation problems," International Journal of Systems Science, vol. 46, no. $11,2015$.
[18] E. Ali and M. Tavana, "A novel method for solving linear programming problems with symmetric trapezoidal fuzzy numbers," Applied Mathematical Modelling, vol. 38, no. 1718, pp. 4388-4395, 2014.

[19] E. Ali, S. J. Ghomi, and S. M. Mirhosseini-Alizamini, "A revisit of numerical approach for solving linear fractional programming problem in a fuzzy environment," Applied Mathematical Modelling, vol. 57, pp. 459-473, 2018.

[20] E. Ali, "Sensitivity analysis in fuzzy number linear programming problems," Mathematical and Computer Modelling, vol. 53, no. 9-10, pp. 1878-1888, 2011.

[21] G. Ramesh and K. Ganesan, "Duality theory for interval linear programming problems," IOSR Journal of Mathematics, vol. 4, no. 4, pp. 39-47, 2012.

[22] L. Kané, M. Konaté, D. Diawara, M. Diallo, and L. Diabaté, "Duality theory for interval linear programming problems," IOSR Journal of Mathematics, vol. 16, no. 4, pp. 01-10, 2020.

[23] M. Ma, M. Friedman, and A. Kandel, "A new fuzzy arithmetic," Fuzzy Sets and Systems, vol. 108, no. 1, pp. 83-90, 1999.

[24] I. Skalna, B. R. B. Gaweł, B. B. J Duda, and J. O. T. PełechPilichowski, "Advances in fuzzy decision making: theory and practice," Studies in Fuzziness and Soft Computing, Vol. 333, Springer International Publishing, Switzerland, 2015.

[25] J. Kaur and K. Amit, "An introduction to fuzzy linear programming problems: theory, methods and applications," Studies in Fuzziness and Soft Computing, Vol. 340, Springer International Publishing, Switzerland, 2016. 